\title{
Renormalization group study of superfluid phase transition: effect of compressibility
}

\author{
Michal Dančð \\ Institute of Experimental Physics SAS, Watsonova 47, 04001 Košice, Slovakia \\ Michal Hnati讯 \\ Institute of Experimental Physics SAS, Watsonova 47, 04001 Košice, Slovakia \\ Bogolyubov Laboratory of Theoretical Physics, Joint Institute for Nuclear Research, 141980 Dubna, Russian Federation and \\ Faculty of Science, Šafárik University, Moyzesova 16, 04001 Košice, Slovakia \\ Tomáš Lučivjanskył \\ Faculty of Science, Šafárik University, Moyzesova 16, 04001 Košice, Slovakia \\ Lukáš Mižišin 8 \\ Institute of Experimental Physics SAS, Watsonova 4\%, 04001 Košice, Slovakia and \\ Bogolyubov Laboratory of Theoretical Physics, Joint Institute for Nuclear Research, 141980 Dubna, Russian Federation
}

(Dated: July 31, 2020)

\begin{abstract}
Dynamic critical behavior in superfluid systems is considered in a presence of external stirring and advecting processes. The latter are generated by means of the Gaussian random velocity ensemble with white-noise character in time variable and self-similar spatial dependence. The main focus of this work is to analyze an effect of compressible modes on the critical behavior. The model is formulated through stochastic Langevin equations, which are then recast into Janssen-De Dominicis response formalism. Employing the field-theoretic perturbative renormalization group method we analyze large-scale properties of the model. Explicit calculations are performed to the leading oneloop approximation in the double $(\varepsilon, y)$ expansion scheme, where $\varepsilon$ is a deviation from the upper critical dimension $d_{c}=4$ and $y$ describes a scaling properties of the velocity ensemble. Altogether five distinct universality classes are expected to be macroscopically observable. In contrast to the incompressible case, we found that compressibility leads to an enhancement and stabilization of non-trivial asymptotic regimes.
\end{abstract}

\section{INTRODUCTION}

Scaling behavior and related concepts arguably provide many fruitful views not only in theoretical physics [1, 2. Though initially scaling came to prominence in the field of high energy physics and critical phenomena, nowadays, many of its applications can be found in such diverse research areas as biology [3, 4, finance [5, population dynamics [1, 6], epidemics spreading [7] and others.

Among the most studied systems in physics, exhibiting scaling behavior, is superfluid phase transition in liquid helium. In this paper we are concerned with a specific aspect of critical dynamics in the vicinity of $\lambda$ point in superfluid helium ${ }^{4} \mathrm{He}$. In the seminal review of Hohenberg-Halperin [8], these authors categorized various dynamical models to which renormalization group methods have been applied. In order to categorize other forms as well they have denoted these models in alphabetical order so that $\mathrm{E}$ designates a symmetric planar magnet, while $\mathrm{F}$ designates asymmetric planar magnet. In more general model $\mathrm{F}$, dynamics is captured by three fields. Two of them, $\psi$ and $\psi^{\dagger}$ correspond to order pa-

\footnotetext{
* michal.danco@gmail.com

$\dagger$ hnatic@saske.sk

$\ddagger$ tomas.lucivjansky@upjs.sk

$\S$ mizisin@theor.jinr.ru
}

rameter and stand for expectation values of microscopic bosonic operators $\hat{\psi}$ and $\hat{\psi}^{\dagger}$. Third field $m$ describes temperature fluctuations in a system. Interactions between fields are determined from the generalized Poisson brackets, whose forms follow from physically motivated considerations [1, 9]. Model E can be interpreted as a simplified version of model $\mathrm{F}$ in which certain temperature dependence has been neglected 9, 10, and physically different variables are empoyed. However, in practical terms this amounts to an appearance of a complex kinetic coefficient and an intermode cubic coupling term [9, 10]. Both models $\mathrm{E}$ and $\mathrm{F}$ have been analyzed predominantly by renormalization group (RG) methods [1, 9,11]. There remains a long-standing issue [1, 9, 12 14, related to determination, which fixed point of the RG flow actually corresponds to a macroscopically observable regime in experiments. This is not only an academic problem as ensuing non-asymptotic effects hinder experimentally measurable quantities. Possible solutions involve (i) search for correct microscopic model for superfluidity [14, 15], (ii) elaborating existing numerical results through multiloop calculations [16 19, or (iii) appropriate generalizations of models [13, 14.

In this work, we follow the third option (iii) by means of inclusion into the model description external velocity fluctuations. In this regard several generalization have already been proposed [13, 20, 21. It has been shown that incompressible hydrodynamic fluctuations contribute sig- 
nificantly to the value of the $\omega_{w}$-index, which controls stability of large-scale regimes 9, 17. This index is related to a RG behavior of a ratio of two kinetic coefficients (in this paper corresponding ratio is related to a parameter $u$ introduced later in Sec. II).

However, the overall conclusions are by no means decisive. Main problem we want to address in this paper is to analyze a presence of solenoidal modes in velocity fluctuations. In particular, we study what new effects in comparison with the incompressible case can be expected and to what extent is critical behavior affected.

Similarly to typical advective problems in fluid dynamics 22 24] we incorporate velocity fluctuation field $\boldsymbol{v}$ by substituting partial time derivative $\partial_{t}$ with a convective derivative of the form $\partial_{t}+(\boldsymbol{v} \cdot \boldsymbol{\nabla})$. From general considerations [25], we expect that presence of external disturbance, e.g. random impurities or turbulent mixing, might lead to completely new types of critical behavior with richer and more exotic properties 2629 .

To fully specify a theoretical setup let us briefly describe the employed model for velocity fluctuations. We assume that velocity $\boldsymbol{v}=\boldsymbol{v}(t, \boldsymbol{x})$ is a random stochastic Gaussian variable with prescribed statistical properties [24, 30]. In the original formulation [31, the velocity field was further taken to be isotropic, incompressible and decorrelated in a time variable. Without loss of generality, we can set the mean value $\langle\boldsymbol{v}\rangle=0$ and take the pair velocity function in the following form

$$
\left\langle v_{i} v_{j}\right\rangle \propto \delta\left(t-t^{\prime}\right) k^{-d-y} T_{i j}
$$

where $k$ is the wave number, $0<y<2$ is a free parameter with the realistic (Kolmogorov) value $y=4 / 3, d$ is a space dimension, and tensor $T_{i j}$ carries information about vectorial character of velocity modes. This model attracted a lot of interest in the past mainly because of insights it offers into the origin of intermittency and anomalous scaling in the fully developed turbulence [24, 30]. Naively, basic premises of such models might be perceived as too crude and unrealistic. Nevertheless, important effects of parity breaking, anisotropy, or compressibility are easily taken into account [30, 32 35]. It turns out that then the phenomenon of intermittency is even more pronounced than in genuine turbulent flow. The recent studies have also pointed out some significant differences between the zero and finite correlation time problems [34, 36, 37] and between the compressible and incompressible cases 38, 39.

Let us point out a crucial difference between critical dynamical models and the model considered in this work. The basic assumption of the former models is a presence of ambient thermal fluctuations. Coupling with thermal bath provides necessary means by which a critical steady state can be maintained. Deviations from thermal equilibrium are considered small that results into variety of relations between different physical quantities [11. A well-known example is the fluctuation-dissipation theorem, which relates two-point correlation function to susceptibility [1, 10]. On the other hand, inclusion of exter- nal velocity fluctuations effectively drives the critical system away from the thermal equilibrium and leads to an effectively non-equilibrium system. Hence, relations like the fluctuation-dissipation theorem cease to hold, and as a consequence, a theoretical analysis becomes more involved.

In relation to this paper, there was recently put forward an intriguing approach to a similar problem. In contrast to the standard approach to critical systems, in which dynamical models are constructed using generalized Poisson brackets or symmetry considerations 9, particular microscopic approach was suggested [14, 40, 41]. The authors have analyzed various aspects of phase transitions in superfluids were analyzed by means of a non-trivial technique of non-equilibrium Green functions [42]. In particular, an implicit assumption common to many critical models related to incompressibility of underlying fluid [43] was put in a question. Relaxing this condition with allowance of compressible modes results into an effective model fully equivalent to model A of critical dynamics 8. This result seems peculiar as model $\mathrm{A}$ is conceivably the simplest dynamical extension of well-known $\varphi^{4}$ model [10, 44]. For onecomponent order parameter takes simple form

$$
\mathcal{S}_{\mathrm{A}}=-\int \mathrm{d}^{d} x\left(\frac{1}{2}(\boldsymbol{\nabla} \varphi)^{2}+\frac{\tau}{2} \varphi^{2}+\frac{g}{4 !} \varphi^{4}\right),
$$

also known in the literature as Landau-Ginzburg-Wilson action functional [1, 10, 44. As experiments are still lacking in this direction, suggested models are still waiting for a decisive affirmation of their relevance for critical dynamics.

The paper is organized as follows. In Sec. III we give a formulation of the problem by means of Langevin equations. These are then rewritten into the field-theoretic model using Janssen-De Dominicis formalism. The resulting action is amenable to the field-theoretic renormalization group analysis, which is carried out in Sec. III. Sec. IV] is devoted to a detailed analysis of fixed points' structure, and Sec. $\mathrm{V}$ is reserved for a calculation of experimentally relevant critical exponents. Concluding remarks are summarized in Sec. VI. Supplementary sections $\mathrm{A}, \mathrm{B}$ and $\mathrm{C}$ contain technical details about divergent parts of Feynman diagrams, lengthy expressions of $\mathrm{RG}$ functions and coordinates of fixed points.

\section{FIELD-THEORETICAL FORMULATION}

Using the standard terminology proposed in [8, model $\mathrm{E}$ of critical dynamics is described by the non-conserved two-component order parameter composed of two (complex) conjugated fields $\psi(t, \boldsymbol{x})$ and $\psi^{\dagger}(t, \boldsymbol{x})$, and a conserved scalar field $m(t, \boldsymbol{x})$. The former can be viewed as macroscopic averages of the Bose-particle field operators, whereas the latter field $m(t, \boldsymbol{x})$ is a certain linear combination of energy and mass density [10] (or a 
normal component of the magnetization in antiferromagnetic materials). Time evolution of the fields is governed [1, 9, 10, by the following set of equations

$$
\begin{aligned}
\partial_{t} \psi & =\lambda_{0} \frac{\delta \mathcal{S}_{s t}}{\delta \psi^{\dagger}}+i \lambda_{0} g_{30} \psi \frac{\delta \mathcal{S}_{s t}}{\delta m}+f_{\psi} \\
\partial_{t} \psi^{\dagger} & =\lambda_{0} \frac{\delta \mathcal{S}_{s t}}{\delta \psi}-i \lambda_{0} g_{30} \psi^{\dagger} \frac{\delta \mathcal{S}_{s t}}{\delta m}+f_{\psi^{\dagger}} \\
\partial_{t} m & =-\lambda_{0} u_{0} \nabla^{2}\left(\frac{\delta \mathcal{S}_{s t}}{\delta m}\right)+i \lambda_{0} g_{30}\left(\psi^{\dagger} \frac{\delta \mathcal{S}_{s t}}{\delta \psi^{\dagger}}-\psi \frac{\delta \mathcal{S}_{\text {st }}}{\delta \psi}\right) \\
& +f_{m}
\end{aligned}
$$

where $\nabla^{2}=\sum_{i=1}^{d} \partial^{2} / \partial x_{i} \partial x_{i}$ is Laplace operator in $d$ dimensional space, $\partial_{t}=\partial / \partial t$ is time derivative, $\lambda_{0}$ is a kinetic coefficient related to diffusive modes. Let us note that when necessary, we write space dimension $d$ explicitly. This is due to a later use of the RG approach. In contrast to action (2) now the static action functional $\mathcal{S}_{\text {st }}$ is given by the following form in the critical region

$$
\mathcal{S}_{\mathrm{st}}=\int \mathrm{d}^{d} x\left(\psi^{\dagger} \nabla^{2} \psi-\frac{1}{2} m^{2}+m h_{0}-\frac{1}{6} g_{10}\left(\psi^{\dagger} \psi\right)^{2}\right),
$$

and substitution $\varphi(\boldsymbol{x}) \rightarrow \varphi(t, \boldsymbol{x})$ is implicitly assumed in Eq. (3)-(5) in terms stemming from variational derivatives for any member from the set $\varphi \in\left\{\psi, \psi^{\dagger}, m\right\}$. Parameters $g_{10}$ and $g_{30}$ play a role of coupling constants of the theory [1, 10]. Random forces $f_{\psi}, f_{\psi^{\dagger}}$ and $f_{m}$ are assumed to be Gaussian random variables with zero means and correlators $D_{\psi}, D_{\psi^{\dagger}}$ and $D_{m}$ with the white noise character in a time variable. In the time-momentum representation they are given by the following formulas

$$
\begin{aligned}
D_{\psi^{\dagger}}\left(p, t, t^{\prime}\right) & =D_{\psi}\left(p, t, t^{\prime}\right)=\lambda_{0} \delta\left(t-t^{\prime}\right), \\
D_{m}\left(p, t, t^{\prime}\right) & =\lambda_{0} u_{0} p^{2} \delta\left(t-t^{\prime}\right) .
\end{aligned}
$$

Parameter $u_{0}$ is dimensionless and has been introduced for future convenience. Here and below, the bare (unrenormalized) parameters in the renormalization group sense are denoted with the subscript " 0 ". The normalization in relations (7)-(8) has been chosen in such a way that the steady-state equal-time correlation functions of the stochastic problem are calculable exactly with the Boltzmann factor $\exp \left(\mathcal{S}_{\text {st }}\right)$. The stochastic problem 3 . 5), (7), and (8) can be concisely reformulated by means of De Dominicis-Janssen functional formalism [45, 46. Ensuing field-theoretic action of model E [1, 9, 10] then directly follows

$$
\begin{aligned}
\mathcal{S}_{\mathrm{E}} & =2 \lambda_{0} \psi^{\dagger^{\prime}} \psi^{\prime}-\lambda_{0} u_{0} m^{\prime} \nabla^{2} m^{\prime}+\psi^{\dagger^{\prime}}\left\{-\partial_{t} \psi+\lambda_{0}\left[\nabla^{2} \psi\right.\right. \\
& \left.\left.-g_{10}\left(\psi^{\dagger} \psi\right) \psi / 3\right]+i \lambda_{0} g_{30} \psi[-m+h]\right\}+ \text { H. c. } \\
& +m^{\prime}\left\{-\partial_{t} m-\lambda_{0} u_{0} \nabla^{2}[-m+h]\right. \\
& \left.+i \lambda_{0} g_{30}\left[\psi^{\dagger} \nabla^{2} \psi-\psi \nabla^{2} \psi^{\dagger}\right]\right\}
\end{aligned}
$$

Abbreviation H. c. stands for a Hermitian conjugate part of the action with respect to the $\psi$-field. In action (9) we have employed a condensed notation, in which integrals over space-time are implicitly included. For instance, the second term in (9) is an abbreviated form of the expression $m^{\prime} \partial^{2} m^{\prime}=\int \mathrm{d} t \int \mathrm{d}^{d} x m^{\prime}(t, \boldsymbol{x}) \boldsymbol{\nabla}^{2} m^{\prime}(t, \boldsymbol{x})$. Prime fields $\psi^{\prime}$ and $\psi^{\dagger^{\prime}}$ correspond to auxiliary MartinSiggia-Rose response fields [47]. A functional formulation effectively means that the statistical averages of the random quantities in the original stochastic problem (345) can be represented by functional integrals over the full set of fields with the weight functional $\exp \left(\mathcal{S}_{\mathrm{E}}\right)$. In quantum-field-theory terminology various correlation functions then correspond to Green functions of the field theoretic model with action (9). Such formulation is especially convenient for the further use of field-theoretical methods such as Feynman diagrammatic technique and perturbative renormalization group, which provide main theoretical tools in this work.

The next step consists in an introduction of the velocity fluctuations into a theoretical model. According to a standard approach [23, 30, 43 it is sufficient to replace the partial time derivative $\partial_{t}$ by the Lagrangian derivative $\partial_{t}+(\boldsymbol{v} \cdot \boldsymbol{\nabla})$. However, in presence of compressibility this is not sufficient [48, and the following substitutions are necessary

$$
\begin{aligned}
\partial_{t} \psi & \rightarrow \partial_{t} \psi+(\boldsymbol{v} \cdot \boldsymbol{\nabla}) \psi+a_{10} \psi(\boldsymbol{\nabla} \cdot \boldsymbol{v}), \\
\partial_{t} m & \rightarrow \partial_{t} m+(\boldsymbol{v} \cdot \boldsymbol{\nabla}) m+a_{20} m(\boldsymbol{\nabla} \cdot \boldsymbol{v}) .
\end{aligned}
$$

Without inclusion of terms proportional to parameters $a_{10}$ and $a_{20}$ the model ceases to be multiplicatively renormalizable.

In this work we employ the Kraichnan rapid-change model [30, 31, 39] with compressibility of the fluid taken into account. Accordingly, the velocity field $\boldsymbol{v}$ is assumed to be a random Gaussian variable with prescribed statistical properties. By a proper substitution we can always achieve that $\langle\boldsymbol{v}\rangle=0$. Due to the Gaussian character of $\boldsymbol{v}$ the only needed information lies in a specification of the two-point correlation function, which assumes the following form

$$
\left\langle v_{i}(t, \boldsymbol{x}) v_{j}\left(t^{\prime}, \boldsymbol{x}^{\prime}\right)\right\rangle=\delta\left(t-t^{\prime}\right) D_{i j}\left(\boldsymbol{x}-\boldsymbol{x}^{\prime}\right),
$$

where Dirac delta function ensures the Galilean invariance of model 23]. Due to the translational invariance of the flow it is convenient [30 to specify the kernel function $D_{i j}$ in Eq. 12 in the Fourier representation

$$
D_{i j}(\boldsymbol{r})=D_{0} \int \frac{\mathrm{d}^{d} k}{(2 \pi)^{d}} \frac{\theta\left(k-l_{v}\right)}{k^{d+y}}\left[P_{i j}(\boldsymbol{k})+\alpha Q_{i j}(\boldsymbol{k})\right] \mathrm{e}^{i \boldsymbol{k} \cdot \boldsymbol{r}},
$$

where $P_{i j}(\boldsymbol{k})=\delta_{i j}-k_{i} k_{j} / k^{2}$ and $Q_{i j}(\boldsymbol{k})=k_{i} k_{j} / k^{2}$ are the transverse and the longitudinal projector, respectively. Further, $k=|\boldsymbol{k}|$ is the wave number, $D_{0}>0$ is an amplitude factor and $\alpha \geq 0$ is an arbitrary parameter, which might be interpreted as a degree of compressibility in the system [34, 49]. Heaviside function $\theta(x)$ ensures infrared (IR) cutoff of the theory that does not violate Galilean invariance. Momentum IR scale $l_{v}$ is related to 
the external scale of velocity fluctuations $L$ crudely as $l_{v} \sim 1 / L$, but precise form is unimportant for the later discussion.

The case $\alpha=0$ corresponds to the incompressible fluid $(\boldsymbol{\nabla} \cdot \boldsymbol{v}=0)$, whereas $\alpha>0$ describes a deviation from the incompressibility. After a proper rescaling, the limit $\alpha \rightarrow \infty$ at fixed $\alpha D_{0}$ yields purely potential velocity field. The exponent $0<y<2$ is a free parameter that might be interpreted as the Hölder exponent, which expresses a roughness of the velocity field. The Kolmogorov regime corresponds to the value $y=4 / 3$, whereas the Batchelor limit (smooth velocity) is obtained in the limit $y \rightarrow 2$.

The action functional describing statistics of the velocity field $\boldsymbol{v}$ is simply given by a quadratic form

$$
\mathcal{S}_{\mathrm{vel}}=-\frac{1}{2} v_{i} D_{i j}^{-1} v_{j}
$$

where $D_{i j}^{-1}$ is the kernel of the inverse linear operator in Eq. 13. This yields a propagator $\Delta_{v v}$, which in the time - momentum representation takes the following form

$$
\Delta_{v v}(t, \boldsymbol{k})=w_{0} \lambda_{0} \delta(t) \frac{P_{i j}(\boldsymbol{k})+\alpha Q_{i j}(\boldsymbol{k})}{k^{d+y}} .
$$

For convenience, the factor $D_{0}$ from the kernel (13) has been expressed in the following way $D_{0}=w_{0} \lambda_{0}$, so that that RG constants might depend only on $w_{0}$.

To summarize, the total dynamic functional for model $\mathrm{E}$ with an inclusion of external velocity fluctuations is given by a sum of expressions (9) and (14), i.e.,

$$
\mathcal{S}=\mathcal{S}_{\mathrm{E}}+\mathcal{S}_{\mathrm{vel}}
$$

Model $\sqrt{16}$ is amenable to the standard Feynman diagrammatic technique, which is based on the graphical interpretation of linear (solvable) part of the action and non-linear terms therein [1, 10. In graphical means interaction terms are represented by vertices, which are connected by lines. The latter correspond to propagators of the free theory, which are given by the quadratic part of the action. Propagators are conveniently given in the frequency-momentum representation

$$
\begin{aligned}
\Delta_{m m} & =\frac{2 \lambda_{0} u_{0} k^{2}}{\omega^{2}+\lambda_{0}^{2} u_{0}^{2} k^{4}} \theta\left(k-l_{m}\right), \\
\Delta_{m m^{\prime}} & =\frac{1}{-i \omega+\lambda_{0} u_{0} k^{2}}, \\
\Delta_{\psi^{\prime} \psi^{\dagger}} & =\Delta_{\psi^{\dagger^{\prime}} \psi}=\frac{1}{i \omega+\lambda_{0} k^{2}}, \\
\Delta_{\psi \psi^{\dagger^{\prime}}} & =\Delta_{\psi^{\dagger} \psi^{\prime}}=\frac{1}{-i \omega+\lambda_{0} k^{2}}, \\
\Delta_{\psi \psi^{\dagger}} & =\Delta_{\psi^{\dagger} \psi}=\frac{2 \lambda_{0}}{\omega^{2}+\lambda_{0}^{2} k^{4}} \theta\left(k-l_{\psi}\right),
\end{aligned}
$$

where $l_{m}$ and $l_{\psi}$ are IR cutoff scales for fields $m$ and $\psi$. For practical reasons we assume

$$
l_{v}=l_{m}=l_{\psi} \equiv l
$$

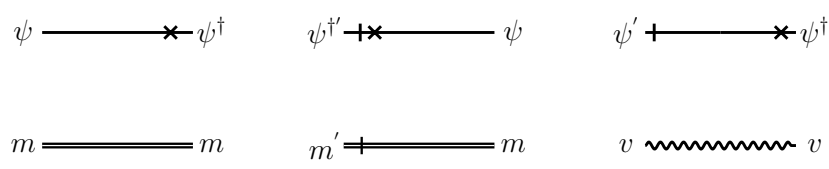

FIG. 1: A graphical representation of the free part of the action (16) that corresponds to lines in the

Feynman diagrammatic technique.

in actual evaluations of Feynman diagrams. This choice can be adopted as univesal quantities do not depend on a particular choice of IR regularization [10, 44].

With every interaction vertex, the algebraic factor

$$
V_{N}\left(x_{1}, \ldots, x_{N} ; \varphi\right)=\frac{\delta^{N} \mathcal{S}[\varphi]}{\delta \varphi\left(x_{1}\right) \ldots \delta \varphi\left(x_{N}\right)}
$$

is associated [10], and $\varphi$ is any field of the theory, i.e. $\varphi \in \Phi$, where

$$
\Phi=\left\{\psi, \psi^{\prime}, \psi^{\dagger}, \psi^{\dagger^{\prime}}, m, m^{\prime}, v\right\}
$$

Here, we readily find three vertex factors $V_{\psi^{\dagger} \psi^{\dagger} \psi \psi}$, $V_{\psi^{\dagger} \psi m}, V_{m^{\prime} \psi^{\dagger} \psi}$ plus their complex conjugates. Their explicit form can be easily inferred from action (9) and in the frequency-momentum representation, it explicitly reads

$$
\begin{aligned}
V_{\psi^{\dagger^{\prime}} \psi^{\dagger} \psi \psi} & =-\frac{2 g_{10} \lambda_{0}}{3} \\
V_{\psi^{\dagger^{\prime}} \psi m} & =-\lambda_{0} g_{30}, \\
V_{m^{\prime} \psi^{\dagger}(\boldsymbol{k}) \psi(\boldsymbol{q})} & =i \lambda_{0} g_{30}\left[\boldsymbol{k}^{2}-\boldsymbol{q}^{2}\right] .
\end{aligned}
$$

The last vertex factor displays a nontrivial dependence on inflowing momenta of fields $\psi^{\dagger}$ and $\psi$.

In addition, set of propagators (17)-19 has to be supplemented with the velocity propagator $\Delta_{v v}$ defined through the relations $(12)$ and $(13)$, respectively. Novel interaction vertices arise from the convective terms (10)(11) as well. Their vertex factors are

$$
\begin{aligned}
& V_{\psi^{\dagger^{\prime}} \psi(\boldsymbol{k}) v_{i}(\boldsymbol{q})}=V_{\psi^{\prime} \psi^{\dagger}(\boldsymbol{k}) v_{i}(\boldsymbol{q})}=i k_{i}+i a_{10} q_{i}, \\
& V_{m^{\prime} m(\boldsymbol{k}) v_{i}(\boldsymbol{q})}=i k_{i}+i a_{20} q_{i} .
\end{aligned}
$$

Let us recall that the parameter $y$ is not related to the spatial dimension and can be varied independently. For the RG analysis of the full-scale problem it is important that all the interactions become logarithmic simultaneously. Otherwise, one of them would be IR irrelevant with respect to the other and it should be discarded 10, 44. As a result, some of the scaling regimes of the full model would be lost. Instead of the ordinary $\varepsilon$ expansion in the single-charge models, the coordinates of the fixed points, critical dimensions and other quantities are now calculable in double expansion scheme $(\varepsilon, y)$.

The perturbation theory of the model is amenable to the standard Feynman diagrammatic expansion [10, 44, 50. A starting point of the perturbation theory is a 


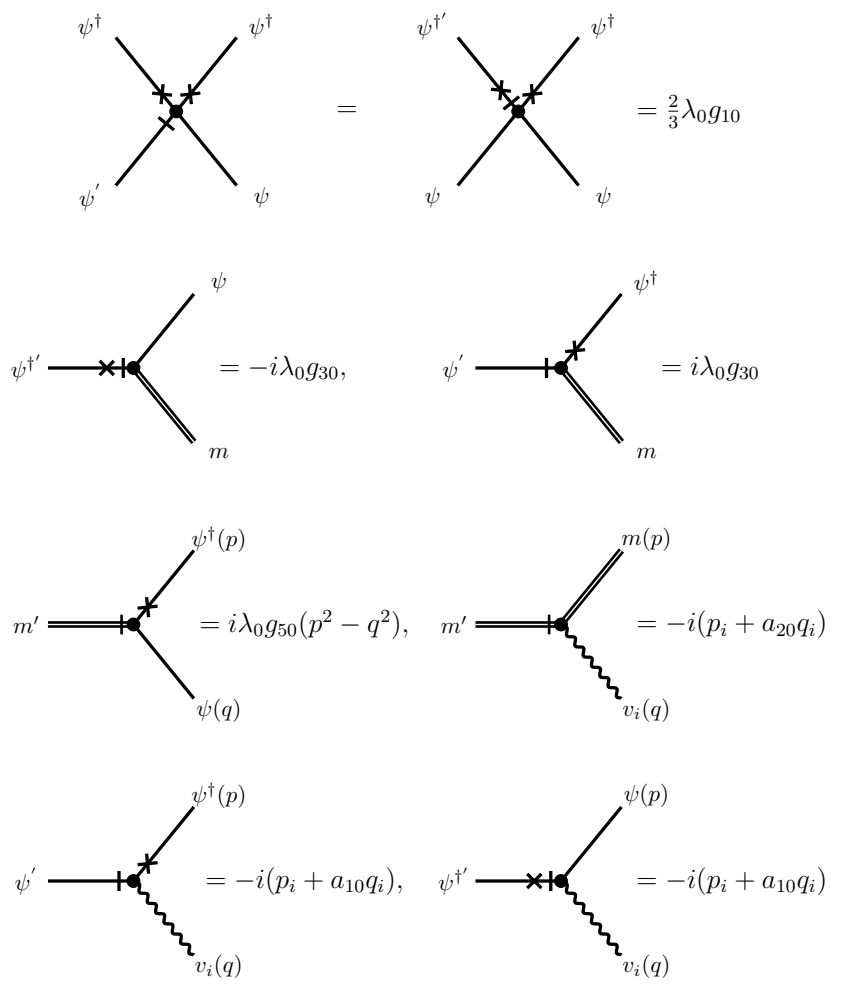

FIG. 2: A graphical representation of the nonlinear part of the action (16) that corresponds to interaction vertices in the Feynman diagrammatic technique.

free part of the action (16). By graphical means, it is represented as lines in the Feynman diagrams, whereas the non-linear terms in (16) correspond to vertices connected by the lines. The bare propagators are graphically depicted in Fig. 1, and interaction vertices in Fig. 2.

\section{RENORMALIZATION GROUP ANALYSIS}

A standard goal in statistical physics lies in determination of macroscopic (large-scale) behavior of the system. The RG procedure allows to exploit scale invariance at the critical point and an elimination of UV divergences yields an information about the IR behavior [10, 44]. There are different prescriptions for the renormalization procedure.

In contrast to the usual situations in critical models, here we deal with a model exhibiting two small parameters $\varepsilon$ and $y$. Similarly, it occured in various context in the past 51 53. Due to presence of two formally small expansion parameters, the RG approach differs somewhat from an usual formalism. First, we assume that the model is regularized by means of an analytic regularization augmented with a dimensional regularization. As has been elucidated [54, 55] mostly used MS scheme suffers from potential deficiencies and is thus not satisfactory from a theoretical point of view. Instead, for calculations of RG constants, we choose a normalization point scheme. Because we restrict ourselves here to the leading one-loop approximation, only two types of UV singularities arise: we find either a pole of type $1 / \varepsilon$ or $1 / y$, respectively. Such simple structure pertains only to the lowest orders in a perturbation scheme. In higher loop-approximations, poles in the form of general linear combinations in $\varepsilon$ and $y$ are expected to arise. Moreover, non-trivial issues related to vector character of fields are expected [56].

\section{A. Canonical dimensions}

A starting point of the RG approach is an analysis of canonical dimensions. Dynamical models of type (9), in contrast to static models, demonstrate two-scale behavior. This accounts for an assignment of two independent (momentum and frequency) canonical dimensions to each quantity $F$ (a field or a parameter in the action functional). Further, since we work with the translationally invariant theory it is sufficient to analyze only one-particle-irreducible (1PI) functions of the model.

The momentum dimension $d_{F}^{k}$ and the frequency dimension $d_{F}^{\omega}$ are determined from the standard normalization conditions

$$
\begin{array}{ll}
d_{k}^{k}=-d_{x}^{k}=1, & d_{\omega}^{k}=d_{t}^{k}=0, \\
d_{k}^{\omega}=d_{x}^{\omega}=0, & d_{\omega}^{\omega}=-d_{t}^{\omega}=1,
\end{array}
$$

and from the requirement that each term in the action functional has to be a dimensionless quantity [10, 44. Then, based on $d_{F}^{k}$ and $d_{F}^{\omega}$ one can introduce a total canonical dimension $d_{F}=d_{F}^{k}+2 d_{F}^{\omega}$, (in the free theory time derivative $\partial_{t}$ should scale in the same way as Laplace operator $\partial^{2}$ ). The dimensions of all quantities appear-

\begin{tabular}{|c|c|c|c|c|c|c|}
\hline$Q$ & $p, 1 / x$ & $\omega, 1 / t$ & $\psi, \psi^{\dagger}$ & $\psi^{\prime}, \psi^{\dagger^{\prime}}$ & $m, m^{\prime}, h$ & $v$ \\
\hline \hline$d_{Q}^{p}$ & 1 & 0 & $\frac{d}{2}-1$ & $\frac{d}{2}+1$ & $\frac{d}{2}$ & -1 \\
\hline$d_{Q}^{\omega}$ & 0 & 1 & 0 & 0 & 0 & 1 \\
\hline$d_{Q}$ & 1 & 2 & $\frac{d}{2}-1$ & $\frac{d}{2}+1$ & $\frac{d}{2}$ & 1 \\
\hline \hline$Q$ & $\lambda_{0}$ & $u_{0}$ & $g_{10}$ & $g_{30}, g_{50}$ & $w_{0}$ & $a_{10}, a_{20}, \alpha$ \\
\hline \hline$d_{Q}^{p}$ & -2 & 0 & $\varepsilon$ & $\frac{\varepsilon}{2}$ & $y$ & 0 \\
\hline$d_{Q}^{\omega}$ & 1 & 0 & 0 & 0 & 0 & 0 \\
\hline$d_{F}$ & 0 & 0 & $\varepsilon$ & $\frac{\varepsilon}{2}$ & $y$ & 0 \\
\hline
\end{tabular}

TABLE I: Canonical dimensions of the fields and parameters for model $\mathrm{E}$ with activated hydrodynamic modes defined by action (16).

ing in action functional $\mathcal{S}$ are summarized in Tab. I It follows that the model is logarithmic (the coupling constants become dimensionless) when $4-d=0(\varepsilon=0)$ and $y=0$. The total canonical dimension of an arbitrary 1PI function [10] is given by the relation

$$
d_{\Gamma}=d+2-\sum_{\varphi \in \Phi} N_{\varphi} d_{\varphi}
$$


where the sum runs over a set of all fields $\Phi$ (defined in (23)) appearing in a given 1PI function $\Gamma$. The total dimension $d_{\Gamma}$ is a formal index of the UV divergence. Due to the compressibility, the derivative $\partial$ on the external line $m^{\prime}$ in graphs of the 1-irreducible functions can not be singled out. It follows that the formal and real UV exponent are the same $d_{\Gamma}=\delta_{\Gamma}$. Superficial UV divergences, whose removal requires counterterms, can be present only in those functions $\Gamma$ for which $d_{\Gamma}$ is a nonnegative integer [10. It is easy to verify that all needed counterterms have a form of various terms already contained in action (9). By inspection of the graphs we observe that all nontrivial diagrams in the term $m^{\prime} \partial_{t} m$ vanish. In 20] it has been demonstrated that the linkage to critical statics is violated due to the inclusion of the velocity field, but the multiplicative renormalization can be recovered by considering a new charge associated with the interaction term $m^{\prime}\left(\psi^{\dagger} \nabla^{2} \psi-\psi \boldsymbol{\nabla}^{2} \psi^{\dagger}\right)$. More precisely, instead of writing this term with the charge $g_{3}$ (see Eq. (5.149) in Chapter 5.20 in [10]), it has to be given as follows

$$
g_{5} m^{\prime}\left(\psi^{\dagger} \nabla^{2} \psi-\psi \nabla^{2} \psi^{\dagger}\right),
$$

where in general a new charge $g_{5}$ does not coincide with the charge $g_{3}$, i.e. $g_{5} \neq g_{3}$. In summary, the fieldtheoretic renormalized action for model $\mathrm{E}$ with velocity fluctuations in condensed notation takes the following form

$$
\begin{aligned}
\mathcal{S}_{\mathrm{R}} & =2 Z_{1} \lambda \psi^{\dagger^{\prime}} \psi^{\prime}-Z_{2} \lambda u m^{\prime} \partial^{2} m^{\prime}-\frac{1}{2} v D^{-1} v \\
& +\psi^{\dagger^{\prime}}\left\{-Z_{3} \nabla_{t} \psi-Z_{4} a_{1}(\boldsymbol{\nabla} \cdot \boldsymbol{v}) \psi+\lambda\left[Z_{5} \partial^{2} \psi\right.\right. \\
& \left.\left.-Z_{6} g_{1} \mu^{\varepsilon}\left(\psi^{\dagger} \psi\right) \psi / 3\right]+Z_{7} i \lambda g_{3} \mu^{\varepsilon / 2} \psi[-m+h]\right\}+ \text { H. c. } \\
& +m^{\prime}\left\{-Z_{8} \nabla_{t} m-Z_{9} a_{2}(\boldsymbol{\nabla} \cdot \boldsymbol{v}) m-\lambda u \partial^{2}\left[-Z_{10} m\right.\right. \\
& \left.+h]+i \lambda g_{5} \mu^{\varepsilon / 2} Z_{11}\left[\psi^{\dagger} \partial^{2} \psi-\psi \partial^{2} \psi^{\dagger}\right]\right\}
\end{aligned}
$$

where $\lambda, u, g_{1}, g_{3}, g_{5}, a_{1}, a_{2}$ are renormalized analogs of the bare parameters (written with the subscript " 0 "), $Z_{i}, i=1, \ldots, 11$ are renormalization constants and $\mu$ is the renormalization mass [10, 44. A full specification of employed normalization conditions reads

$$
\begin{aligned}
\left.\Gamma_{\psi^{+}{ }^{\prime}{ }^{\prime}}\right|_{*} & =2 \lambda, \\
\left.\frac{\partial \Gamma_{m^{\prime} m^{\prime}}}{\partial k^{2}}\right|_{*} & =\lambda u \\
\left.\frac{\partial \Gamma_{\psi^{+}{ }^{\prime} \psi}}{\partial(i \Omega)}\right|_{*} & =-\left.\frac{1}{\lambda} \frac{\partial \Gamma_{\psi^{+{ }^{\prime} \psi}}}{\partial \boldsymbol{k}^{2}}\right|_{*}=1, \\
\left.\frac{\partial \Gamma_{m^{\prime} m}}{\partial(i \Omega)}\right|_{*} & =-\left.\frac{1}{\lambda u} \frac{\partial \Gamma_{m^{\prime} m}}{\partial \boldsymbol{k}^{2}}\right|_{*}=1, \\
\left.\frac{\partial \Gamma_{m^{\prime} \psi^{+}(\boldsymbol{p}) \psi(\boldsymbol{q})}}{\partial \boldsymbol{p}^{2}}\right|_{*} & =-\left.\frac{\partial \Gamma_{m^{\prime} \psi^{+}(\boldsymbol{p}) \psi(\boldsymbol{q})}}{\partial \boldsymbol{q}^{2}}\right|_{*}=i \lambda g_{5}, \\
\left.\Gamma_{\psi^{+\prime} \psi^{+} \psi \psi}\right|_{*} & =-\frac{3 \lambda g_{1}}{2}
\end{aligned}
$$

$$
\begin{gathered}
\left.\frac{\partial \Gamma_{\psi^{+{ }^{\prime}} \psi(\boldsymbol{p}) v_{j}(\boldsymbol{q})}}{\partial p_{j}}\right|_{*}=\left.\frac{1}{a_{1}} \frac{\partial \Gamma_{\psi^{+{ }^{\prime}} \psi(\boldsymbol{p}) v_{j}(\boldsymbol{q})}}{\partial q_{j}}\right|_{*}=-i, \\
\left.\frac{\partial \Gamma_{m^{\prime} m(\boldsymbol{p}) v_{j}(\boldsymbol{q})}}{\partial p_{j}}\right|_{*}=\left.\frac{1}{a_{2}} \frac{\partial \Gamma_{m^{\prime} m(\boldsymbol{p}) v_{j}(\boldsymbol{q})}}{\partial q_{j}}\right|_{*}=-i .
\end{gathered}
$$

For convenience, we have introduced $*$ coordinates specified as follows

$$
\Omega_{i}=0, \quad k_{i}=0, \quad \mu=l,
$$

where index $i$ enumerates independent external frequency or momenta entering given the 1PI function and the IR scale $l$ was introduced in Eq. 222.

The unrenormalized $\mathcal{S}$ and the renormalized action functional $\mathcal{S}_{\mathrm{R}}$ are related by the standard formula

$$
\mathcal{S}_{\mathrm{R}}(\Phi)=\mathcal{S}\left(Z_{\Phi} \Phi\right) .
$$

Direct conseuqence of this formula are multiplicative relations for the fields $Z_{\Phi} \Phi=\left\{Z_{\varphi} \varphi: \varphi \in \Phi\right\}$, and parameters [10, 44]

$$
\begin{array}{rlrlrl}
\lambda_{0} & =\lambda Z_{\lambda}, & u_{0} & =u Z_{u}, & & g_{10}=g_{1} \mu^{\varepsilon} Z_{g_{1}}, \\
a_{10} & =a_{1} Z_{a_{1}}, & a_{20} & =a_{2} Z_{a_{2}}, & & w_{0}=w \mu^{y} Z_{w}, \\
g_{30} & =g_{3} \mu^{\varepsilon / 2} Z_{g_{3}}, & g_{50} & =g_{5} \mu^{\varepsilon / 2} Z_{g_{5}} . &
\end{array}
$$

Since the term $\mathcal{S}_{\text {vel }}(\boldsymbol{v})$ given by (14) is non-local in the spatial variable, we know that according to general rules of the RG technique [10] it should not be renormalized. The parameter $\alpha$ is not renormalized at all, i.e. $\alpha_{0}=\alpha$, and serves as a free parameter of the theory. Due to the Galilean symmetry ensured by the presence of $\delta$-function in correlator (12), both terms in the Lagrangian derivative $\nabla_{t}$ are renormalized with the same renormalization constants. In addition, the quadratic term $v D^{-1} v / 2$ in the action (32) is not renormalized because of a passive nature of the advecting fields. As a direct consequence the velocity field $\boldsymbol{v}$ is not renormalized and two relations follow

$$
Z_{w} Z_{\lambda}=1, \quad Z_{\alpha}=Z_{v}=1 .
$$

In the leading one-loop approximation, the 1irreducible two-point Green functions take the form

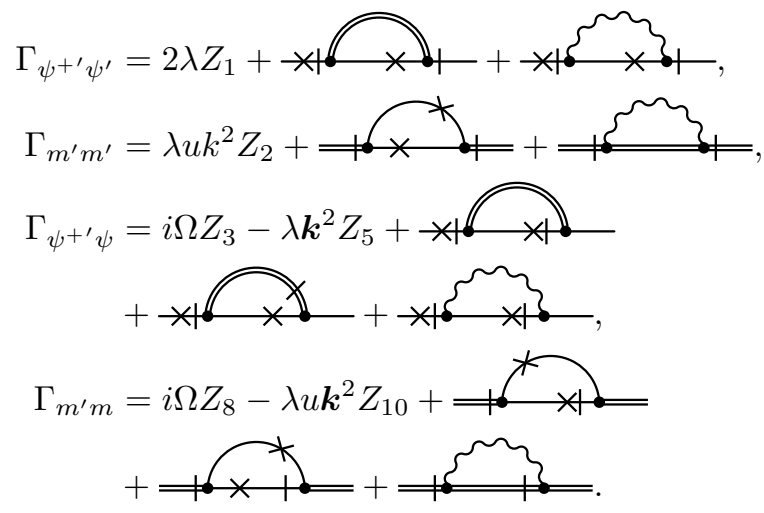

Let us note that due to the structure of the vertex factors the relation $Z_{m^{\prime}} Z_{m}=1$ is fulfilled. 
Further, the 1-irreducible Green functions accounting for non-linearities can be graphically represented as follows
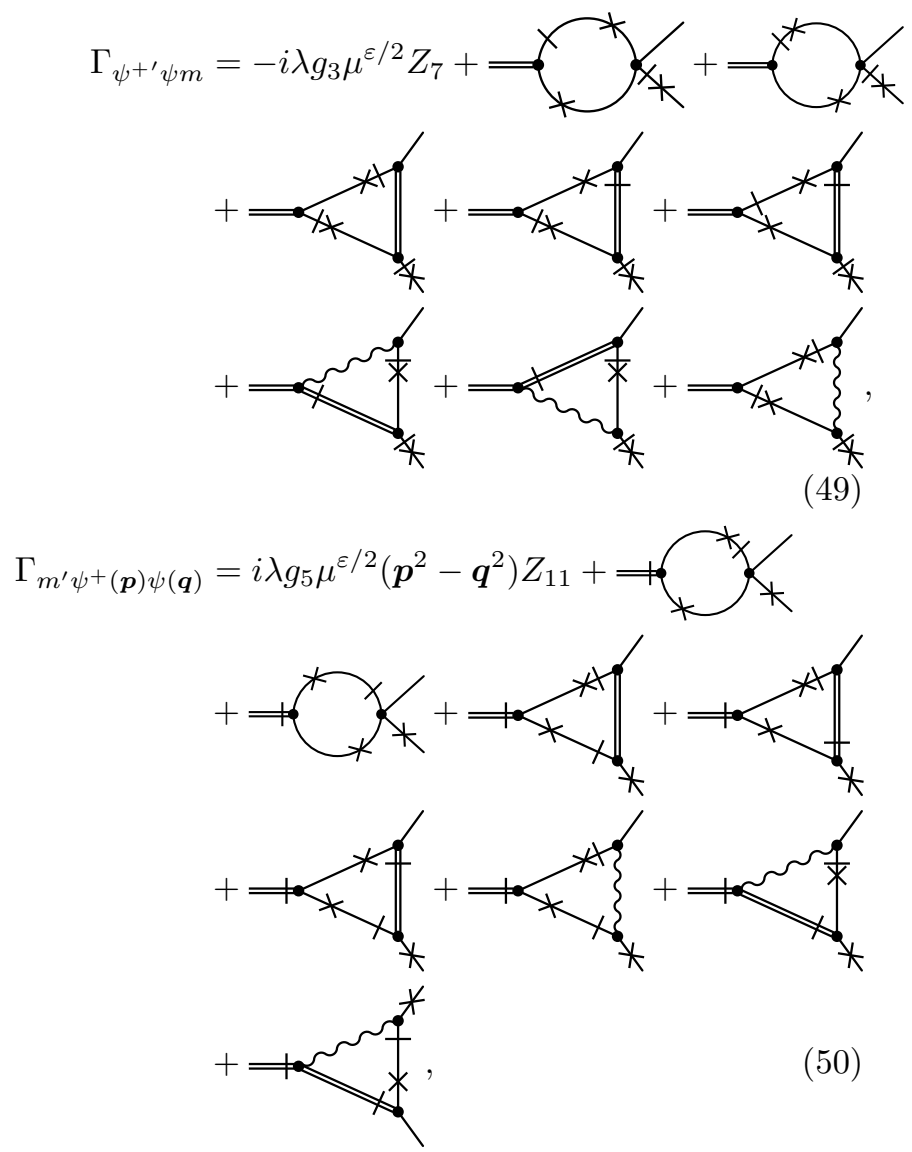

$\Gamma_{\psi^{+} \psi^{+} \psi \psi}=-\frac{2}{3} \lambda g_{1} \mu^{\varepsilon} Z_{6}+$
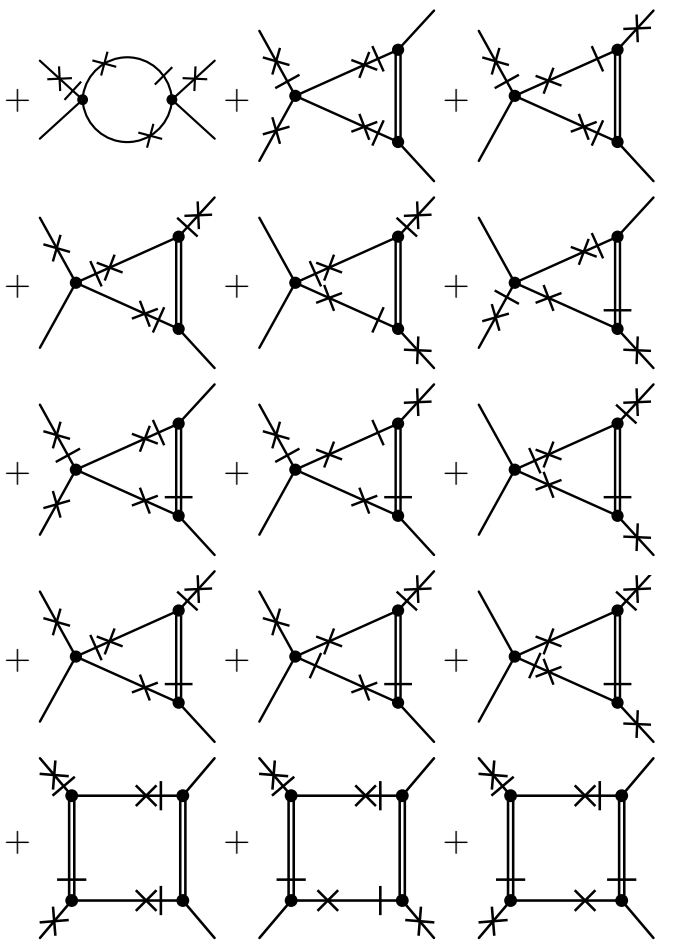
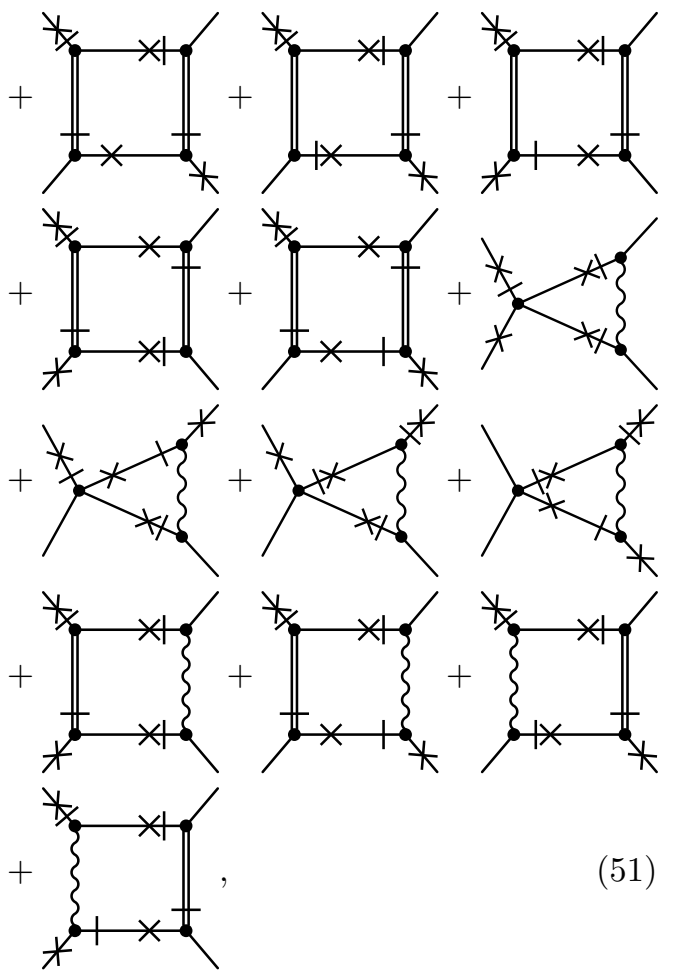

$\Gamma_{\psi^{+} \psi(\boldsymbol{p}) v_{j}(\boldsymbol{q})}=-i p_{j} Z_{3}-i a_{1} q_{j} Z_{4}+\cdots$
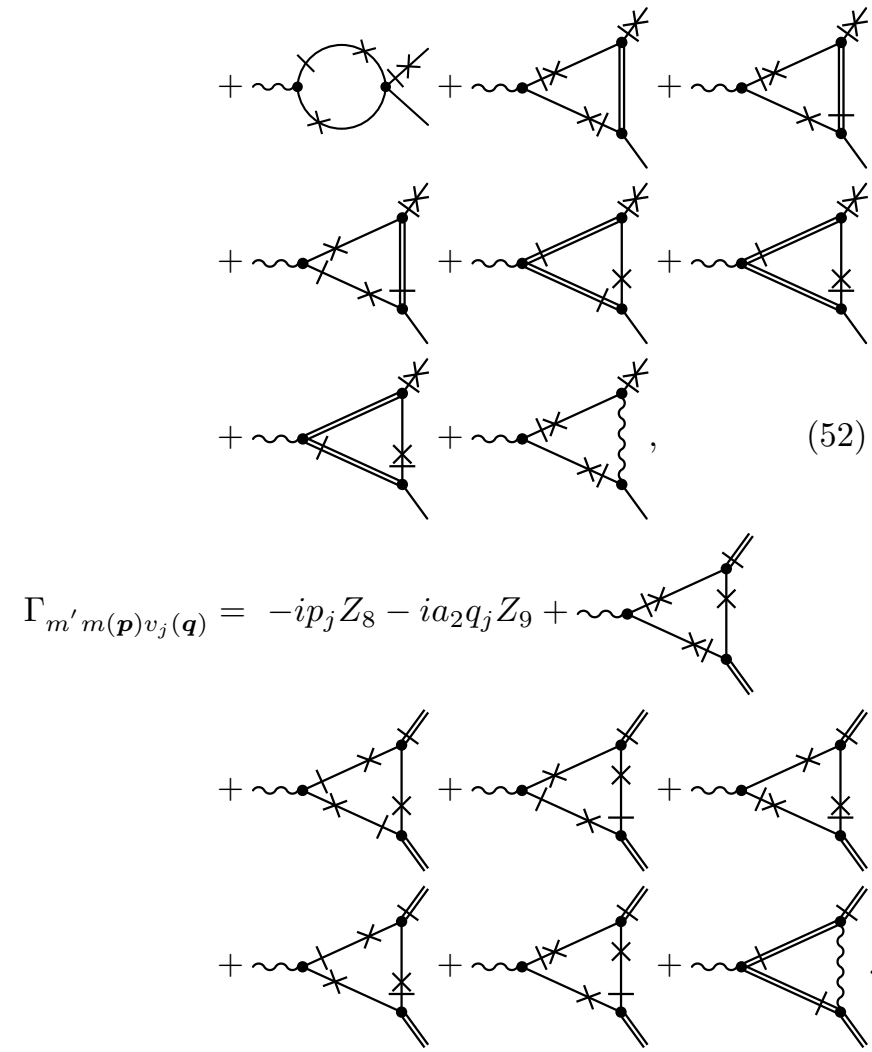

(53)

A major reduction of divergent diagrams comes from two observations. First, according to general rules of critical dynamics [10] any Feynman graph constructed solely 
from retarded propagators does not possess UV divergence. Second, a structure of interaction vertices allows, in some cases, to pull out momentum dependence and thus reduce the effective dimension of internal momentum integration. This then also leads to UV convergence of the corresponding Feynman diagram.

A calculation in the employed RG scheme proceeds in a standard fashion and we have summarized all the results in Appendix A. Note that prefactors contain explicit $d$-dependence stemming from vector and tensorial character of interactions.

Technical difficulties related to the chosen IR cutoff were circumvented by a proper extraction of external momentum from a given diagram. Once correct frequency or momentum dependence was pulled out of a diagram, it was permissible to set all external frequencies and momenta inside integral to zero. This greatly simplifies calculations of divergent parts of Feynman diagrams. However, we expect this to be much more cumbersome in twoand higher-loop approximations.

\section{B. RG functions}

RG invariance [10, 44] can be conveniently expressed by the differential equation $\mathcal{D}_{\mu} \Gamma=0$, where the differentiation with respect to renormalization mass $\mu$ in the operator $\mathcal{D}_{\mu}=\mu \partial_{\mu}$ is performed at fixed values of (bare) unrenormalized variables. For the 1PI renormalized Green function, it can be rewritten as $\left[\mathcal{D}_{R G}-n_{\Phi} \gamma_{\Phi}\right] \Gamma_{R}(\mu, \ldots)=$ 0 , where $n_{\Phi}$ is the number of fields of a given renormalized 1PI Green function. The operator $\mathcal{D}_{R G}$ stands for $\mathcal{D}_{\mu}$ in terms of the renormalized variables

$$
\left.\mathcal{D}_{R G} \equiv \mu \frac{\partial}{\partial \mu}\right|_{0}=\mu \frac{\partial}{\partial \mu}+\sum_{g_{i} \in g} \beta_{g_{i}} \frac{\partial}{\partial g_{i}}-\gamma_{\lambda} \lambda \frac{\partial}{\partial \lambda},
$$

where the summation runs over all charges of the theory. For convenience we have introduced compact notation

$$
g \equiv\left\{g_{1}, g_{3}, g_{5}, u, w, a_{1}, a_{2}\right\}
$$

The differentiation in (54) at fixed values of the bare parameters is indicated explicitly by the subscript " 0 ". The beta functions $\beta_{g_{i}}$ and anomalous dimensions $\gamma_{F}$ of the mode are defined by the logarithmic derivatives [10, 44

$$
\beta_{i}=\left.\frac{\partial g_{i}}{\partial \ln \mu}\right|_{0}, \quad \gamma_{F}=\left.\frac{\partial \ln Z_{F}}{\partial \ln \mu}\right|_{0} .
$$

straightforward application of these definitions onto the relations 43] yields

$$
\begin{array}{ll}
\beta_{g_{1}}=g_{1}\left(-\varepsilon-\gamma_{g_{1}}\right), & \beta_{g_{3}}=g_{3}\left(-\frac{\varepsilon}{2}-\gamma_{g_{3}}\right), \\
\beta_{g_{5}}=g_{5}\left(-\frac{\varepsilon}{2}-\gamma_{g_{5}}\right), & \beta_{w}=w\left(-y-\gamma_{w}\right), \\
\beta_{a_{1}}=-a_{1} \gamma_{a_{1}}, & \beta_{a_{2}}=-a_{2} \gamma_{a_{2}}
\end{array}
$$

$$
\beta_{u}=-u \gamma_{u}, \quad \beta_{\alpha}=-\alpha \gamma_{\alpha} .
$$

In particular, the $\beta_{\alpha}$ function identically vanishes due to the aforementioned constraint $Z_{\alpha}=1$. To obtain the remaining renormalization constants in the MS scheme, the UV-divergent terms (poles in $\varepsilon$ and $y$ in our case) have to be extracted from the loop expansion of the corresponding 1PI functions. Renormalization constants $Z_{i}, i=1, \ldots, 11$ are related to the renormalization constants of the parameters and fields by means of relations

$$
\begin{aligned}
Z_{\lambda} & =Z_{w}^{-1}=\frac{Z_{5}}{Z_{3}}, & Z_{u} & =\frac{Z_{10} Z_{3}}{Z_{5} Z_{8}}, \\
Z_{g_{5}} & =\frac{Z_{11} Z_{1}}{Z_{5}^{2}}\left(\frac{Z_{10}}{Z_{2} Z_{8}}\right)^{1 / 2}, & Z_{g_{1}} & =\frac{Z_{6} Z_{1}}{Z_{5}^{2} Z_{3}}, \\
Z_{g_{3}} & =\frac{Z_{7}}{Z_{5}}\left(\frac{Z_{2}}{Z_{10} Z_{8}}\right)^{1 / 2}, & Z_{a_{1}} & =\frac{Z_{4}}{Z_{3}}, \\
Z_{\psi^{\prime}} & =\left(\frac{Z_{1} Z_{3}}{Z_{5}}\right)^{1 / 2}, & Z_{\psi} & =\left(\frac{Z_{3} Z_{5}}{Z_{1}}\right)^{1 / 2}, \\
Z_{m} & =\left(\frac{Z_{10} Z_{8}}{Z_{2}}\right)^{1 / 2}, & Z_{a_{2}} & =\frac{Z_{9}}{Z_{8}}, \\
Z_{m^{\prime}} & =\left(\frac{Z_{2} Z_{8}}{Z_{10}}\right)^{1 / 2}, & &
\end{aligned}
$$

where we have used two additional relations [10] for fields renormalization

$$
Z_{\psi^{\prime}}=Z_{\psi^{\dagger^{\prime}}}, \quad Z_{\psi^{\dagger}}=Z_{\psi}
$$

From the second relation in (56) anomalous dimensions $\gamma_{F}$ can be directly obtained from the renormalization constants (61)-(65). A special feature of the one-loop approximation is the fact that to this order we have found

$$
Z_{8}=Z_{9}=1 \text {. }
$$

Substituting 69 in 65 leads to

$$
Z_{a_{2}}=1 \text {. }
$$

Hence, parameter $a_{2}$ can be also regarded as a free parameter of the model to the order of perturbation theory.

\section{SCALING REGIMES AND THE FIXED-POINT STRUCTURE}

From an experimental point of view, most relevant for statistical physics is the IR-asymptotic behavior, i.e. behavior of Green functions at small frequencies $\omega \rightarrow 0$ and momenta $\boldsymbol{k} \rightarrow 0$. This is related to large-scale macroscopic regimes of a given renormalizable field theoretic model, which are associated with IR attractive fixed points of the corresponding RG equations [10, 44]. A fixed point (FP) is defined as such point $g^{*} \equiv\left\{g_{1}^{*}, g_{3}^{*}, g_{5}^{*}, u^{*}, a_{1}^{*}, a_{2}^{*}, w^{*}\right\}$ for which all $\beta$ functions simultaneously vanish, i.e.

$$
\beta_{g_{i}}\left(g^{*}\right)=0
$$


where $g_{i}$ is any member of the set $g$ defined in Eq. 55. The IR stability of a given fixed point is determined by the matrix of first derivatives of $\beta$ functions

$$
\Omega_{i j}=\left.\frac{\partial \beta_{i}}{\partial g_{j}}\right|_{g^{*}} .
$$

Coordinates of fixed points do not possess a direct physical information, because they depend on the chosen renormalization scheme [10, 44]. However, in perturbation theory universal quantities and number of fixed points actually depend on the chosen RG scheme [55]. In order to proceed in actual calculations, we have expanded prefactors containing $d$-parameter in Feynman diagrams by $d=4-\varepsilon$ (see Appendix A for details). Though not correct in higher loop calculations, in the one-loop approximation this can be regarded as an adequate operation.

For completeness, we list all found fixed points in Appendix C

For IR attractive fixed points all real parts of eigenvalues of matrix 72 are positive. Moreover, physical conditions $u^{*}>0$ and $w^{*}>0$ have to be fulfilled, which is due to their appearance in free pair correlation functions (see Eq. (17) and Eq. (15)).

A thorough analysis of beta functions has revealed that there are several possible regimes in case without thermal fluctuations, i.e., regimes with a fixed point coordinate $g_{3}^{*}=0$. It is worth mentioning that for a purely transverse velocity field $(\alpha=0)$ the terms containing $a_{10}, a_{20}$ in (10)-11 and all subsequent expressions vanish, and such parameters disappear from the model.

An actual analysis proved to be rather cumbersome and not very illuminating. The main technical problems were related to an appearance of parameters $a_{1}, a_{2}$ brought about by compressibility of velocity field in action (32). Moreover, compressibility parameter $\alpha$ is free from any restriction and can attain any positive value 34. Thus when possible, we therefore try to present our findings by graphical means. Technical details can be found in the Appendices.

Altogether eight fixed points have been found. However, only some of them are IR stable. A trivial Gaussianlike fixed point FP1 is IR-stable in the region restricted by inequalities

$$
\varepsilon<0, \quad y<0 .
$$

FP1 corresponds to a free model, for which all interactions are irrelevant and is stable above upper critical space dimension $d>d_{c}=4$. The corresponding critical exponents attain their mean-field values.

The fixed points FP2, FP3 and FP4 are unstable because the corresponding eigenvalues of the $\Omega$ matrix $(72)$ (see Tab. V) always contain one positive and one negative eigenvalue for any value of exponent $\varepsilon$.

For FP5 fixed points' values of charges $g_{1}, g_{3}$ and $g_{5}$ vanish identically. The nonzero coordinates $w$ and $u$ hint at IR relevance of the turbulent advection. This regime corresponds to a well-known passive advection problem [24, 34]. We recall that parameters $\alpha, a_{1}$ and $a_{2}$ should be regarded as free parameters and stability regions might exhibit a non-trivial dependence on them. This point is IR-stable in the region restricted effectively by two inequalities. First one is simply the condition $y>0$ and the second restriction is given by one of the following inequalities,

$$
\begin{aligned}
{\left[3-\alpha\left(3 a_{1}^{2}-3 a_{1}+1\right)\right] y } & >\varepsilon \frac{3+\alpha}{4}, \\
{\left[3-\alpha\left(8 a_{1}^{2}-4 a_{1}+1\right)\right] y } & >\varepsilon \frac{3+\alpha}{2}, \\
{\left[3-\alpha\left(4 a_{1} a_{2}-1\right)\right] y } & >\varepsilon \frac{3+\alpha}{2} .
\end{aligned}
$$

Which one of them will be imposed depends on the value of free parameters $\alpha, a_{1}$ and $a_{2}$. The corresponding inequality for arbitrary value of parameters can be found in Tab. [II] Parameter values corresponding to the endpoints in Tab. II lead to inequalities of the same form. A thorough analysis of FP5 reveals some interesting features. Increasing one of the free parameters with two remaining parameters fixed, boundaries of stability region shift. From a technical point of view, this is caused by a form of the left hand side of inequalities (74)-(76). For instance, we choose $a_{1}=1 / 4$ and $a_{2}$ from the interval $\left(0, \frac{3}{2}\right)$. Then, whenever the compressibility parameter $\alpha$ attains a value smaller than $\alpha^{*}=8$, the region of stability (Fig. 3b, 4 , $5 \mathrm{a}$ is restricted by inequality 75 . On the other hand, for $\alpha$ larger than $\alpha^{*}$, restrictions comes from inequality (74). For a special case $\alpha^{*}=8$, the inequalities (74) and 75) are actually of the same form (Fig. 5b). For $\alpha=6$ the inequality 775 takes a simple form of $\varepsilon<0$. With an increasing value of $\alpha$, the boundary of FP5 rotates in the counterclockwise direction, and in the purely potential limit $\alpha \rightarrow \infty$ it approaches the ray $y=-\frac{4}{7} \varepsilon$. Let us note that in a one-loop approximation boundaries of stability regions between different fixed points are often given by straight lines. In a higher-loop calculations overlapping regions might appear.

The remaining three regimes FP6, FP7, and FP8 are possible candidates for new regimes, since for them both velocity and self-interactions of model $\mathrm{E}$ are IR relevant. Fixed points FP5 and FP6 differ only in IR-relevance of the self-interaction term $m^{\prime}\left(\psi^{\dagger} \partial^{2} \psi-\psi \partial^{2} \psi^{\dagger}\right)$, which is irrelevant for the former and relevant for the latter. Similarly to the previous case of FP5, the stability region of FP6 is affected by values of free parameters $a_{1}, a_{2}$ and $\alpha$. However, FP6 is realizable only for certain intervals, which can be summarized as follows

$$
\begin{aligned}
& a_{1} \in\left(0, \frac{\sqrt{3}-1}{2}\right), \\
& \alpha \in\left(\frac{3}{1-2 a_{1}-2 a_{1}^{2}}, \infty\right), \\
& a_{2} \in\left(0, \frac{3(\alpha-1)}{4 a_{1} \alpha}+\frac{3\left(a_{1}-1\right)}{2}\right) .
\end{aligned}
$$




\begin{tabular}{|c|c|c|c|}
\hline$a_{1}$ & $\alpha$ & $a_{2}$ & Ineq. \\
\hline \hline$\left(0, \frac{\sqrt{3}-1}{2}\right)$ & $\left(0, \alpha^{*}\right)$ & $\left(0, \frac{4 a_{1}^{2}-2 a_{1}+1}{2 a_{1}}\right)$ & 750 \\
\hline$\left(0, \frac{\sqrt{3}-1}{2}\right)$ & $\left(0, \alpha^{*}\right)$ & $\left(\frac{4 a_{1}^{2}-2 a_{1}+1}{2 a_{1}}, \infty\right)$ & 76 \\
\hline$\left(0, \frac{\sqrt{3}-1}{2}\right)$ & $\left(\alpha^{*}, \infty\right)$ & $\left(0, \frac{3 \alpha\left(2 a_{1}^{2}-2 a_{1}+1\right)-3}{4 a_{1} \alpha}\right)$ & 74 \\
\hline$\left(0, \frac{\sqrt{3}-1}{2}\right)$ & $\left(\alpha^{*}, \infty\right)$ & $\left(\frac{3 \alpha\left(2 a_{1}^{2}-2 a_{1}+1\right)-3}{4 a_{1} \alpha}, \infty\right)$ & 76 \\
\hline$\left(\frac{\sqrt{3}-1}{2}, \infty\right)$ & $(0, \infty)$ & $\left(0, \frac{4 a_{1}^{2}-2 a_{1}+1}{2 a_{1}}\right)$ & 750 \\
\hline$\left(\frac{\sqrt{3}-1}{2}, \infty\right)$ & $(0, \infty)$ & $\left(\frac{4 a_{1}^{2}-2 a_{1}+1}{2 a_{1}}, \infty\right)$ & 760 \\
\hline
\end{tabular}

TABLE II: Intervals of free parameters $a_{1}, \alpha, a_{2}$ with the corresponding inequality restricting the stability region of FP5. Here, $\alpha^{*}$ stands for the expression

$$
3 /\left(1-2 a_{1}-2 a_{1}^{2}\right) \text {. }
$$

The necessary condition for FP6 being stable is $y>0$. Further, it is restricted by the inverse inequality (74) as can be easily seen in Tab. V] The second restriction depends on the choice of the parameter $a_{2}$. If the value of $a_{2}$ is smaller than the value $a_{2 b}=(3+\alpha) /\left(4 a_{1} \alpha\right)+$ $\left(5 a_{1}-1\right) / 2$, the second boundary is given by the inequality 75 . For $a_{2}>a_{2 b}$, the boundary is given by the following inequality

$$
\left[9-\alpha\left(1-6 a_{1}+6 a_{1}^{2}+4 a_{1} a_{2}\right)\right] y>\varepsilon(3+\alpha) .
$$

The overall analysis of FP6 can be divided into two cases, whereby each of them corresponds to a different interval of parameters. The situation is qualitatively the same for $a_{1}>1 / 5$ as in previous case of FP5. For $a_{1}<1 / 5$ the region of stability lies in the first quadrant of $(\varepsilon, y)$-plane (see Fig. 6b).

For illustration purposes, the regions of IR stability in the $(\varepsilon, y)$-plane are depicted in Fig. 6. The regime FP6 is stable for the choice $a_{1}=1 / 4, \alpha>8$ and the border is specified by (75) up to $a_{2}=9 / 8+3 / \alpha$, Fig. 6a. With an increasing value of $a_{2}$, the restriction changes to (78) and the stability region shrinks down into a boundary line for $a_{2}=15 / 8-3 / \alpha$. Beyond the value $a_{2}=1+3 / \alpha$ the border of FP5 is specified by the inequality 76 and in the $(\varepsilon, y)$-plane a void region appears between regimes FP5 and FP6.

For the remaining two fixed points FP7 and FP8, the fixed points' coordinate of charge $g_{3}$ is zero. From the results in Appendix $\mathrm{C}$ it can be readily noticed that the main difference between regimes FP5 and FP7 lies in the IR relevance of the quartic interaction terms (proportional to the charge $g_{1}$ ). In addition, the coordinate $a_{1}$ attains a definite value $1 / 4$ for the point FP7, whereas for FP5 it is not fixed.

In contrast to a previously studied case, the stability analysis of FP7 is less involved. First, one can show that free parameters $\alpha$ and $a_{2}$ have to belong to the following intervals

$$
\alpha \in(0,8), \quad a_{2} \in\left(0, \frac{3}{2}\right) .
$$

Further, parameter $y$ has to be strictly positive and further restrictions read

$$
\begin{aligned}
(3+\alpha) \varepsilon & >(6-\alpha) y, \\
(48-7 \alpha) y & >4(3+\alpha) \varepsilon, \\
2\left[3-\alpha\left(a_{2}-1\right)\right] y & >(3+\alpha) \varepsilon,
\end{aligned}
$$

For small values of the parameter $a_{2}$ the second restriction comes from (81) and it holds up to $a_{2}=15 / 8-3 / \alpha$. Above this value, the boundary is defined by inequality 82 .

Let us note that FP7 is unstable for incompressible case (Fig. 3a), whereas even small (non-zero) values of $\alpha$ leads to its stabilization. With an increasing value of $\alpha$, the region of IR stability rotates counterclockwise (see Fig. 4 and finally shrinks down to a boundary line $y=$ $-11 \varepsilon / 2$ for the limit value of compressibility parameter $\alpha=8$ (see Fig. 5).

For the remaining fixed point $\mathrm{FP} 8$ parameters $\alpha$ and $a_{2}$ are restricted by the following conditions

$$
\alpha \in\left(\frac{8}{5}, \infty\right), \quad a_{2} \in\left(0, \min \left\{\frac{9}{8}+\frac{3}{\alpha}, \frac{15}{8}-\frac{3}{\alpha}\right\}\right) .
$$

The stability region is always bounded by the inequality

$$
\left[72-\alpha\left(8 a_{2}-1\right)\right] y>8(3+\alpha) \varepsilon .
$$

The second restriction is given by 81 with the opposite sign of inequality for $\alpha<8$ and the regime is stable for $a_{2}<15 / 8-3 / \alpha$. On the other hand, for $\alpha>8$, the inequality 80 determines the boundary and FP8 is stable for $a_{2}<9 / 8+3 / \alpha$. With an increasing value of parameter $\alpha$, the region of IR stability rotates counterclockwise. In the potential limit $\alpha \rightarrow \infty$ there always exists stability region whenever $a_{2}<9 / 8$ is fulfilled. Once $a_{2}>9 / 8$ the regime becomes unstable.

It is worth mentioning that we have not found a nontrivial fixed point that would correspond to a case with all the nonlinearities being IR relevant. Notwithstanding this observation, activated velocity fluctuations affect the stability analysis through newly introduced charges $g_{5}, w, a_{1}$, and $a_{2}$. From the practical point of view they contribute to the $\Omega$ matrix $(72)$ with new columns and rows present. Indeed, a comprehensive numerical analysis has revealed that they play an essential role in the fixed points stability.

The same result has been obtained in the case of incompressible fluid [13. We conclude that the presence of compressibility has a stabilizing effect on the regimes where nonlinearities are relevant. The regions of IR stability for these fixed points are shown in Figs. 3.6.

Let us focus on two special cases that correspond to the Kolmogorov spectrum of the velocity $y=4 / 3$ and Batchelor limit $y=2$ (smooth velocity field), respectively. We 

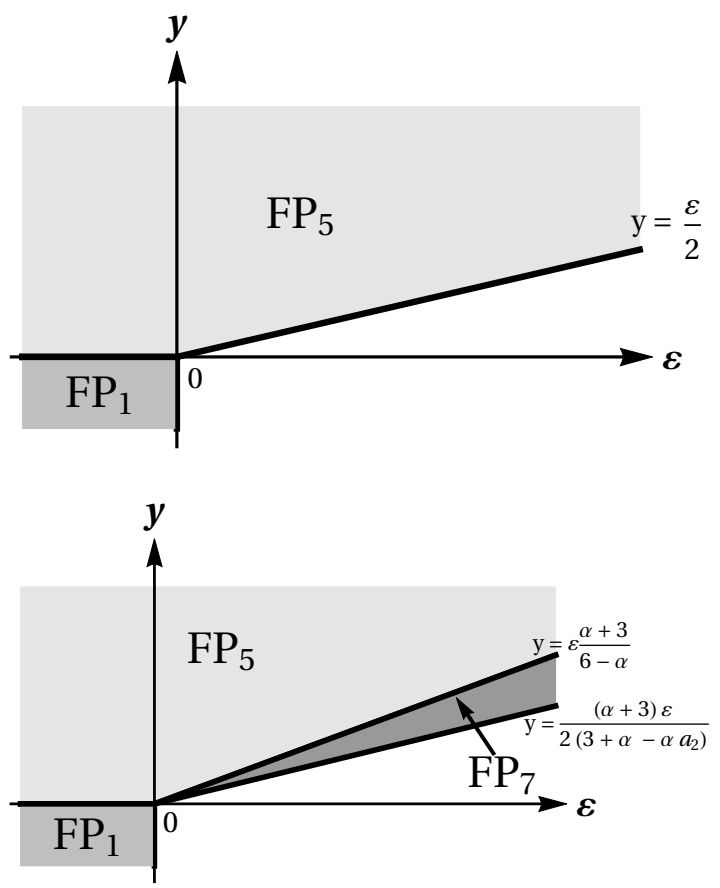

FIG. 3: Regions of stability of the fixed points in the model for compressibility parameter $\alpha=0$ (top) and for the following restricted choice of parameters $a_{1}=1 / 4$, $\alpha \in\left(0, \frac{8}{5}\right)$ and $a_{2} \in\left(0, \frac{3}{2}\right)$ (bottom).
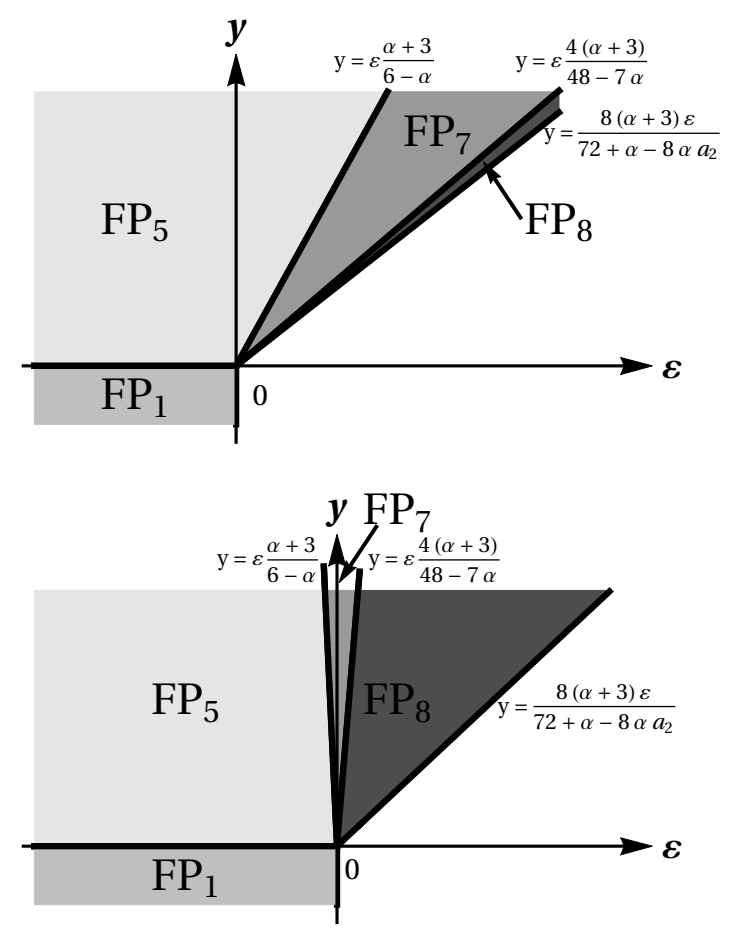

FIG. 4: Stability regions of the fixed points for which $a_{1}=1 / 4$ and for the following restricted choice of parameters $\alpha \in\left(\frac{8}{5}, 6\right), a_{2} \in\left(0, \frac{15}{8}-\frac{3}{\alpha}\right)$ (top) and $\alpha \in\left(6, \frac{48}{7}\right), a_{2} \in\left(0, \frac{15}{8}-\frac{3}{\alpha}\right)$ (bottom).
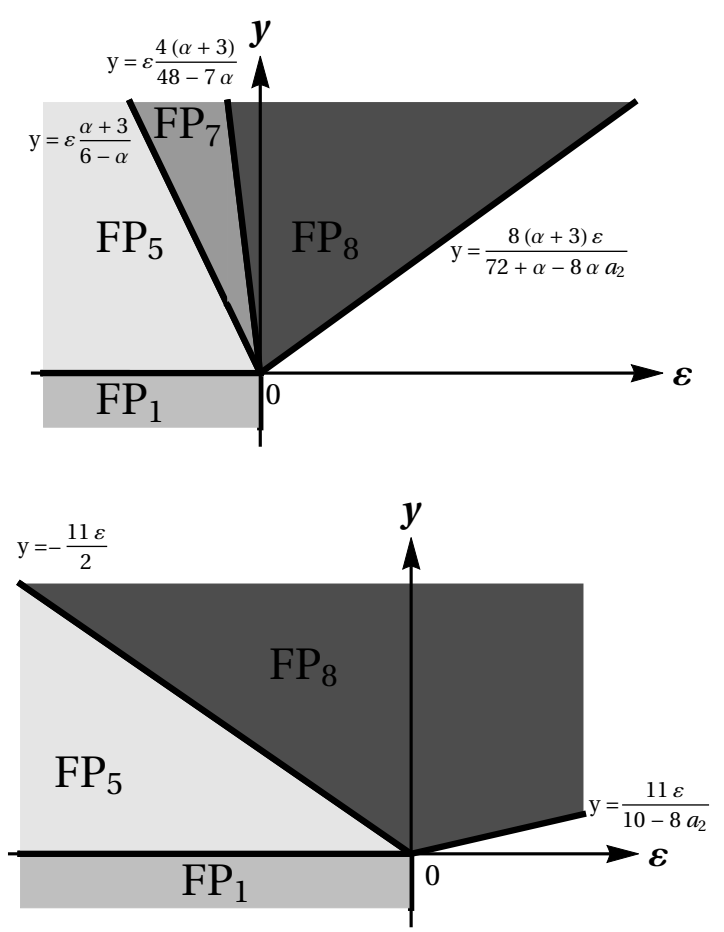

FIG. 5: Stability regions of the fixed points for which $a_{1}=1 / 4$ and for the following restricted choice of parameters $\alpha \in\left(\frac{48}{7}, 8\right), a_{2} \in\left(0, \frac{1}{8}+\frac{9}{\alpha}\right)$ (top) and $\alpha=8, a_{2} \in\left(0, \frac{5}{4}\right)$ (bottom).

can see that three fixed points belong to a given value of scaling parameter $y$ for the real space dimension $d=3$ $(\varepsilon=1)$. The regime FP6 is located in a non-physical region and could not be realized. Further, the analysis is focused on the case $a_{1}=1 / 4$, where the rest of the nontrivial regime is depicted in Fig. 7. For small values of the compressibility parameter $\alpha$ both the Kolmogorov regime and Bachelor limit belong to universality class FP5. As has been already mentioned this regime corresponds to a passively advected scalar without self-interaction and for a small value of $a_{1}, a_{2}, \alpha$, it still can be stable for real scaling parameters $y, \varepsilon$. However, for a larger value of $\alpha$, the Kolmogorov and Batchelor value happen to lie in the stability region (Fig. 7) of the novel non-trivial regime FP7 or FP8.

Nevertheless, we expect that a qualitative picture for large values of compressibility should remain the same. In order to properly describe effects of strong compressibility and to better understand non-universal effects for turbulent mixing one should proceed one step further and employ a more sophisticated model for compressible velocity fluctuations [53, 57, 58].

\section{CRITICAL DIMENSIONS}

Existence of an IR attractive fixed point implies existence of scaling behavior of the Green functions in the IR range. In this critical scaling all the IR irrelevant parameters $(\lambda, \mu$ and the coupling constants) are fixed 

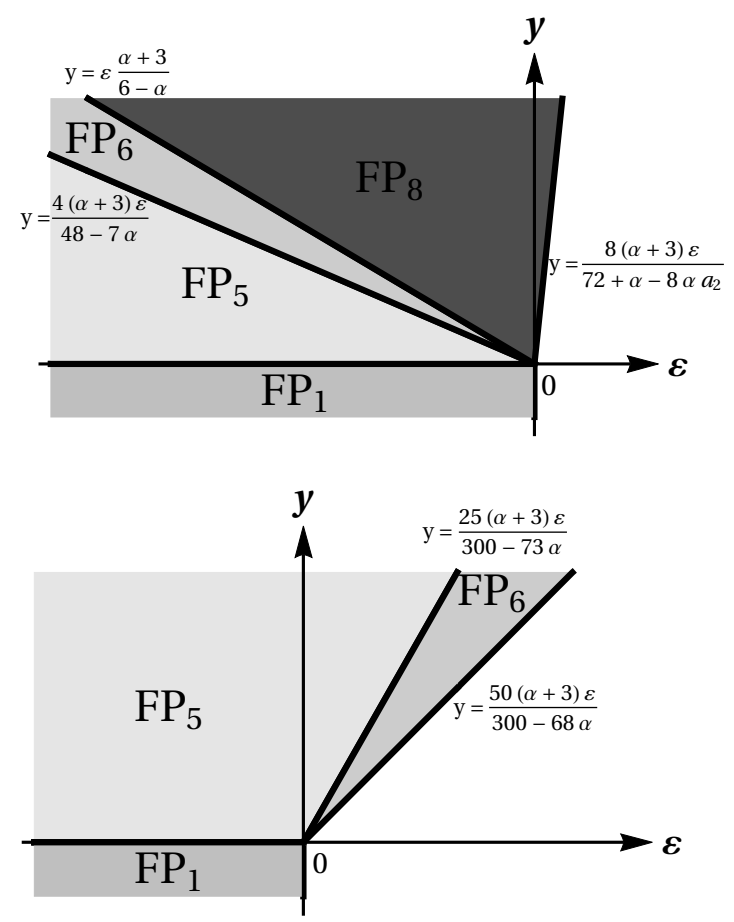

FIG. 6: Stability regions of the fixed points in the model for the following restricted choice of parameters $a_{1}=1 / 4, \alpha>8$ and $a_{2} \in\left(0, \frac{1}{8}+\frac{9}{\alpha}\right)$ (top) and $a_{1}=1 / 10, \alpha \in\left(\frac{50}{13}, \frac{300}{73}\right), a_{2} \in\left(0, \frac{9}{4}+\frac{15}{2 \alpha}\right)$ (bottom).

and the IR relevant parameters (coordinates / momenta, times / frequencies and the fields) are dilated. In the leading IR asymptotic behavior of renormalized Green functions $G^{R}$ satisfy the RG equation 54 with the substitution $g \rightarrow g_{*}$ for the full set of the couplings [10, 50]. This directly yields the fundamental RG equation

$$
\left\{\mathcal{D}_{\mu}-\gamma_{\lambda}^{*} \mathcal{D}_{\lambda}+\sum_{\varphi} N_{\varphi} \gamma_{\varphi}^{*}\right\} G^{R}=0,
$$

where for convenience we write $\mathcal{D}_{x} \equiv x \partial / \partial x, \gamma_{F}^{*}$ is the value of the anomalous dimension at the fixed point, and the summation over all types of the fields $\varphi$ appearing in $G^{R}$ is implied. Equations of this type describe the scaling with dilatation of the variables whose derivatives enter the differential operator [10, 50].

The canonical scale invariance with respect to momentum and frequency variable, respectively, can be expressed by two relations

$$
\left[\sum_{\sigma} d_{\sigma}^{k} \mathcal{D}_{\sigma}-d_{G}^{k}\right] G^{R}=0, \quad\left[\sum_{\sigma} d_{\sigma}^{\omega} \mathcal{D}_{\sigma}-d_{G}^{\omega}\right] G^{R}=0,
$$

where $\sigma$ is the full set of all the arguments of $G^{R}, d_{\sigma}^{k}$ and $d_{\sigma}^{\omega}$ are canonical dimensions of variable $\sigma$ with respect to momentum, and frequency, respectively. In order to derive proper scaling relation with fixed IR irrelevant parameters $\mu$ and $\nu$ one has to combine Eqs. (85) and (86)
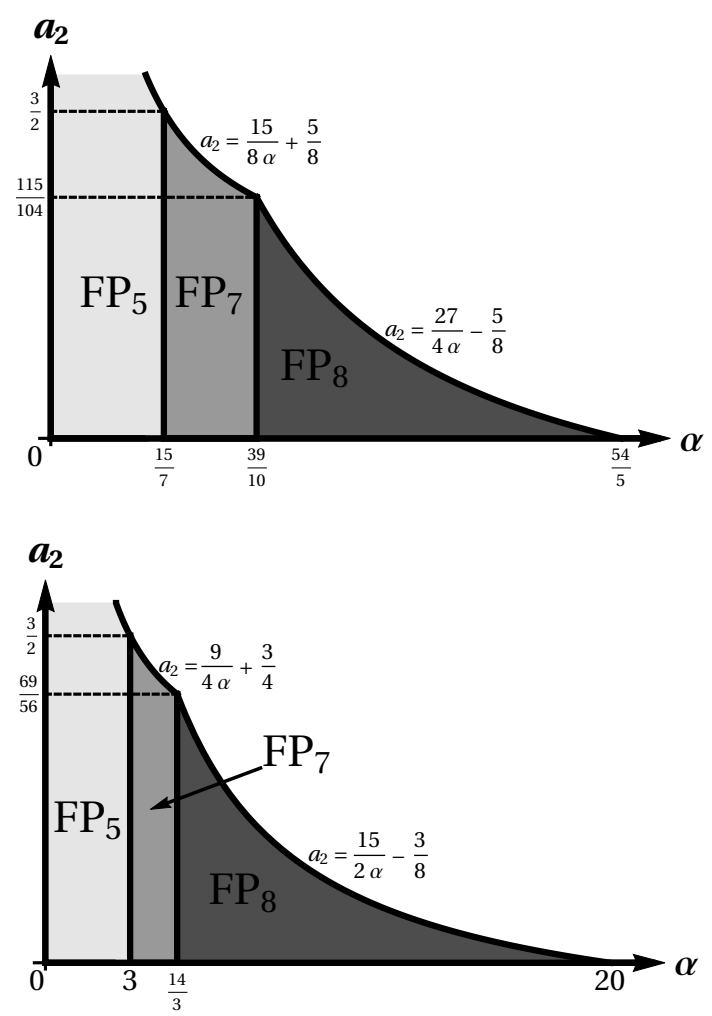

FIG. 7: Stability regions in the plane $\left(\alpha, a_{2}\right)$ in which depicted areas correspond to the fixed points for fixed parameters $\varepsilon=1$ and $a_{1}=1 / 4$. Choice $y=4 / 3$ corresponds to the Kolmogorov spectrum of velocity (top). Choice $y=2$ corresponds to the Batchelor limit (bottom).

in such a way that the derivatives with respect to these parameters are eliminated [10, 23]. This yields an equation of critical IR scaling for the model

$$
\left\{-\mathcal{D}_{\boldsymbol{x}}+\Delta_{t} \mathcal{D}_{t}+\Delta_{\lambda} \mathcal{D}_{\lambda}-\sum_{\varphi} N_{\varphi} \Delta_{\varphi}\right\} G^{R}=0
$$

with the following notation

$$
\Delta_{F}=d_{F}^{k}+\Delta_{\omega} d_{F}^{\omega}+\gamma_{F}^{*}, \quad \Delta_{\omega}=-\Delta_{t}=2-\gamma_{\lambda}^{*} .
$$

Here, $\Delta_{F}$ is the critical dimension of the quantity $F$, while $\Delta_{t}$ and $\Delta_{\omega}$ are the critical dimensions of time and frequency, and $\gamma_{F}^{*}$ is the value of the anomalous dimension of a quantity $F$ at the fixed point. In our case we have obtained critical dimensions for parameters and fields of IR stable fixed points in the following form

- FP1 (Gaussian fixed point)

$$
\begin{aligned}
& \Delta_{\omega}=2, \quad \Delta_{\psi}=\frac{d}{2}-1, \quad \Delta_{\psi^{\prime}}=\frac{d}{2}+1, \\
& \Delta_{m}=\Delta_{m^{\prime}}=\frac{d}{2} .
\end{aligned}
$$


- FP5 (passively advected scalar without selfinteraction)

$$
\begin{aligned}
& \Delta_{\omega}=2-y, \quad \Delta_{\psi}=1-\frac{\varepsilon}{2}+\frac{y}{2}-\frac{2 \alpha y\left(a_{1}-1\right)^{2}}{3+\alpha}, \\
& \Delta_{\psi^{\prime}}=3-\frac{\varepsilon}{2}-\frac{y}{2}+\frac{2 \alpha y\left(a_{1}-1\right)^{2}}{3+\alpha} \\
& \Delta_{m}=\Delta_{m^{\prime}}=2-\frac{\varepsilon}{2} .
\end{aligned}
$$

- FP6 (new non-trivial fixed point)

$$
\begin{aligned}
\Delta_{\omega} & =2-y, \quad \Delta_{\psi}=1-\frac{\varepsilon}{2}+\frac{y}{2}-2 \alpha y \frac{\left(a_{1}-1\right)^{2}}{3+\alpha}, \\
\Delta_{\psi^{\prime}} & =3-\frac{\varepsilon}{2}-\frac{y}{2}+2 \alpha y \frac{\left(a_{1}-1\right)^{2}}{3+\alpha}, \\
\Delta_{m} & =2-\varepsilon+2 y-\alpha y \frac{5+3\left(2 a_{1}-1\right)^{2}}{2(3+\alpha)}, \\
\Delta_{m^{\prime}} & =2-2 y+\alpha y \frac{5+3\left(2 a_{1}-1\right)^{2}}{2(3+\alpha)} .
\end{aligned}
$$

- FP7 (new non-trivial fixed point)

$$
\begin{aligned}
\Delta_{\omega} & =2-y, \quad \Delta_{\psi}=1-\frac{\varepsilon}{2}+\frac{12-5 \alpha}{8(3+\alpha)} y, \\
\Delta_{\psi^{\prime}} & =3-\frac{\varepsilon}{2}-\frac{12-5 \alpha}{8(3+\alpha)} y \\
\Delta_{m} & =\Delta_{m^{\prime}}=2-\frac{\varepsilon}{2} .
\end{aligned}
$$

- FP8 (new non-trivial fixed point)

$$
\begin{aligned}
\Delta_{\omega} & =2-y, \quad \Delta_{\psi}=1-\frac{\varepsilon}{2}+\frac{12-5 \alpha}{8(3+\alpha)} y, \\
\Delta_{\psi^{\prime}} & =3-\frac{\varepsilon}{2}-\frac{12-5 \alpha}{8(3+\alpha)} y, \\
\Delta_{m^{\prime}} & =2-\frac{48-7 \alpha}{8(3+\alpha)} y, \quad \Delta_{m}=2-\varepsilon+\frac{48-7 \alpha}{8(3+\alpha)} y .
\end{aligned}
$$

\section{CONCLUSION}

We have incorporated effects of compressible turbulent mixing and stirring in model $\mathrm{E}$ of critical dynamics. It has been shown how the field-theoretic formulation of such model can be constructed. A multiplicative renormalizability of the ensuing model has been proven, which permits us to employ a field-theoretic perturbative renormalization group. Altogether 62 nontrivial Feynman diagrams have been identified to the leading one-loop approximation. We have found that depending on the values of a spatial dimension $(d=4-\varepsilon)$, a scaling exponent $y$ describing statistics of velocity fluctuations and a degree of compressibility $\alpha$, the model exhibits 5 possible large-scale regimes corresponding to distinct universality classes. Two of them are already well-known: a
Gaussian or trivial fixed point and a passively advected scalar without any self-interaction. The remaining three regimes correspond to novel universality classes for which non-linearities of model $\mathrm{E}$ and turbulent mixing are both relevant. Critical exponents have been calculated and they exhibit dependence on $d, y$ and the compressibility parameter $\alpha$. We have found that compressibility enhances the role of the nonlinear terms in the dynamical equations. The stability region in the $(\varepsilon, y)$ plane, where new nontrivial regimes are stable is thus getting much wider as the degree of compressibility increases. As a result, turbulent mixing becomes more efficient due to combined effects of the mixing and the nonlinear terms.

\section{ACKNOWLEDGMENTS}

The authors thank V. Škultéty for checking part of the calculations in Appendix A and B. The authors are indebted to Nikolay M. Gulitskiy for critical reading of the manuscript. The work was supported by VEGA grant No. 1/0345/17 of the Ministry of Education, Science, Research and Sport of the Slovak Republic, the grant of the Slovak Research and Development Agency under the contract No. APVV-16-0186.

\section{Appendix A: Computation of Feynman diagrams}

In order to simplify notation we have used the following shift

of coupling constants

$$
\frac{e S_{d}}{(2 \pi)^{2}} \rightarrow e
$$

where $S_{d}=2 \pi^{\frac{d}{2}} / \Gamma(d / 2)$ is a convenient geometrical factor, and $e \in\left\{g_{1}, g_{3}^{2}, g_{3} g_{5}, g_{5}^{2}, w\right\}$. In order to avoid any potential ambiguities we explicitly indicate a symmetry coefficient of a given Feynman graph in front of its graphical representation. The assessment and direction of external momenta for 1PI diagrams $m^{\prime} \psi \psi^{\dagger}$ correspond to independent momenta $\boldsymbol{p}$ and $\boldsymbol{q}$ displayed in Fig. 2. There in vertex $\psi^{\dagger} \psi m^{\prime}$, external momenta $\boldsymbol{p}, \boldsymbol{q}$ flow in through fields $\psi^{\dagger}(\boldsymbol{p})$ and $\psi(\boldsymbol{q})$, and flow out through $m^{\prime}(-\boldsymbol{p}-\boldsymbol{q})$. The external momenta are chosen in a way, that they flow only via one internal line at most. Further, for 1PI diagrams $\psi^{\dagger^{\prime}} \psi \boldsymbol{v}$ external momenta $\boldsymbol{p}, \boldsymbol{q}$ flow in as $\psi(\boldsymbol{p})$ and $v_{i}(\boldsymbol{q})$ and then flow out as ${\psi^{+}}^{\prime}(-\boldsymbol{p}-\boldsymbol{q})$, and last for diagrams $m^{\prime} m \boldsymbol{v}$ external momenta $\boldsymbol{p}, \boldsymbol{q}$ flow in as $m(\boldsymbol{p})$ and $v_{i}(\boldsymbol{q})$ and then flow out as $m^{\prime}(-\boldsymbol{p}-\boldsymbol{q})$.

Let us also note that we give results only for diagrams that yield nonzero contributions.

$$
\begin{aligned}
& \text { * }=\frac{2 \lambda g_{3}^{2}}{(1+u) \varepsilon}, \\
& \text { *ศ? } \\
& \frac{1}{2} \underset{\times}{*}=\frac{2 \lambda g_{5}^{2} \boldsymbol{p}^{2}}{d \varepsilon} \text {, }
\end{aligned}
$$




$$
\begin{aligned}
& \frac{1}{2}=\frac{(d-1+\alpha) \lambda w \boldsymbol{p}^{2}}{2 d y} \text {, } \\
& \rightarrow-\left[\frac{i \Omega}{(1+u)^{2}}+\lambda \boldsymbol{p}^{2} \frac{4-d(u+1)}{d(1+u)^{3}}\right] \frac{g_{3}^{2}}{\varepsilon}, \\
& \longrightarrow \frac{g_{3} g_{5}}{\varepsilon}\left[\frac{i \Omega}{(1+u)^{2}}\right. \\
& \left.-\lambda \boldsymbol{p}^{2} \frac{(d(1+u)(2+u)-4)}{d(u+1)^{3}}\right] \text {, } \\
& \rightarrow \text { ? } \\
& \Longrightarrow=-\frac{(d-2) \lambda g_{3} g_{5} \boldsymbol{p}^{2}}{2 d \varepsilon} \\
& +\times=-\frac{(d-2) \lambda g_{3} g_{5} \boldsymbol{p}^{2}}{2 d \varepsilon} \text {, } \\
& =\frac{(d-1+\alpha) \lambda w \boldsymbol{p}^{2}}{2 d y} \\
& \overbrace{*}^{*}=-\frac{i \lambda g_{1} g_{3}}{3 \varepsilon} \text {, } \\
& \overbrace{*}^{*}=\frac{i \lambda g_{1} g_{3}}{3 \varepsilon} \text {, } \\
& =\frac{i \lambda g_{3}^{3}}{(1+u)^{2} \varepsilon} \\
& =\frac{-i \lambda(3+u) g_{3}^{2} g_{5}}{2(1+u)^{2} \varepsilon} \\
& \underbrace{*}_{*}=\frac{i \lambda g_{3}^{2} g_{5}}{2(1+u) \varepsilon} \\
& \underbrace{*}_{*}=-\frac{i \lambda g_{3} \alpha a_{1} a_{2} w}{(1+u) y} \text {, } \\
& \Longrightarrow \gamma_{\star}=\frac{i \lambda g_{5} g_{1}}{3 d \varepsilon}\left[(6-d) \boldsymbol{p}^{2}+(d-2) \boldsymbol{q}^{2}\right. \\
& +4 \boldsymbol{p} \cdot \boldsymbol{q}] \\
& \Longrightarrow \chi_{\star}=-\frac{i \lambda g_{5} g_{1}}{3 d \varepsilon}\left[(6-d) \boldsymbol{p}^{2}+(d-2) \boldsymbol{q}^{2}\right. \\
& +4 \boldsymbol{p} \cdot \boldsymbol{q}]
\end{aligned}
$$
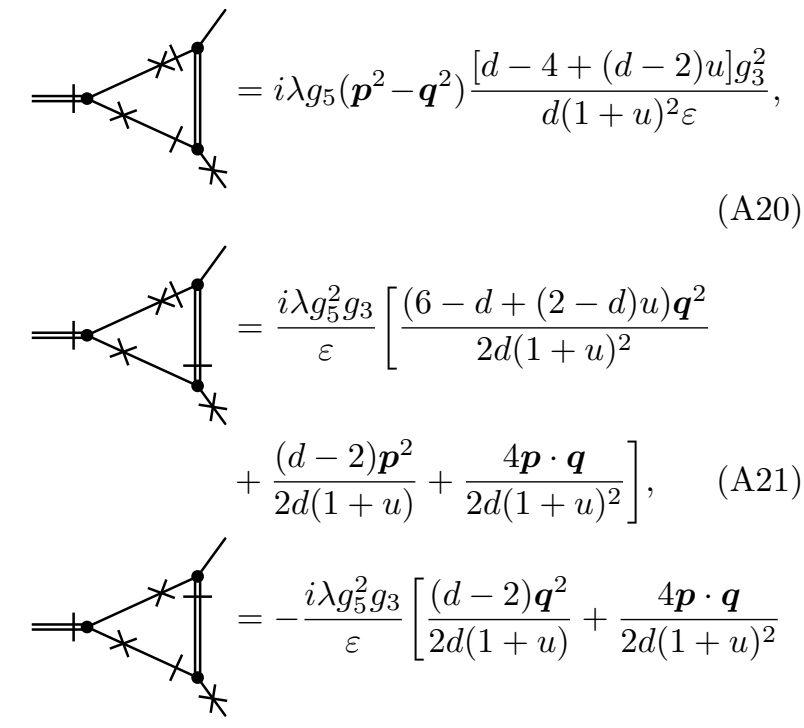$$
\left.+\frac{(6-d+(2-d) u) \boldsymbol{p}^{2}}{2 d(1+u)^{2}}\right]
$$$$
+x_{*}^{*+1}=\left(\boldsymbol{p}^{2}-\boldsymbol{q}^{2}\right) \frac{i \lambda g_{5} \alpha a_{1}\left(2+(d-2) a_{1}\right) w}{2 d y} \text {, }
$$$$
\frac{1}{2}
$$
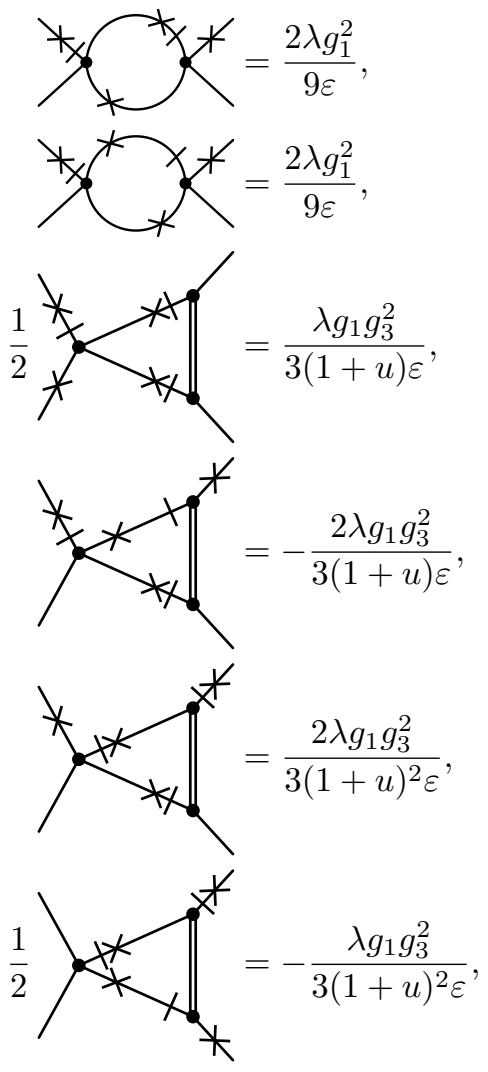

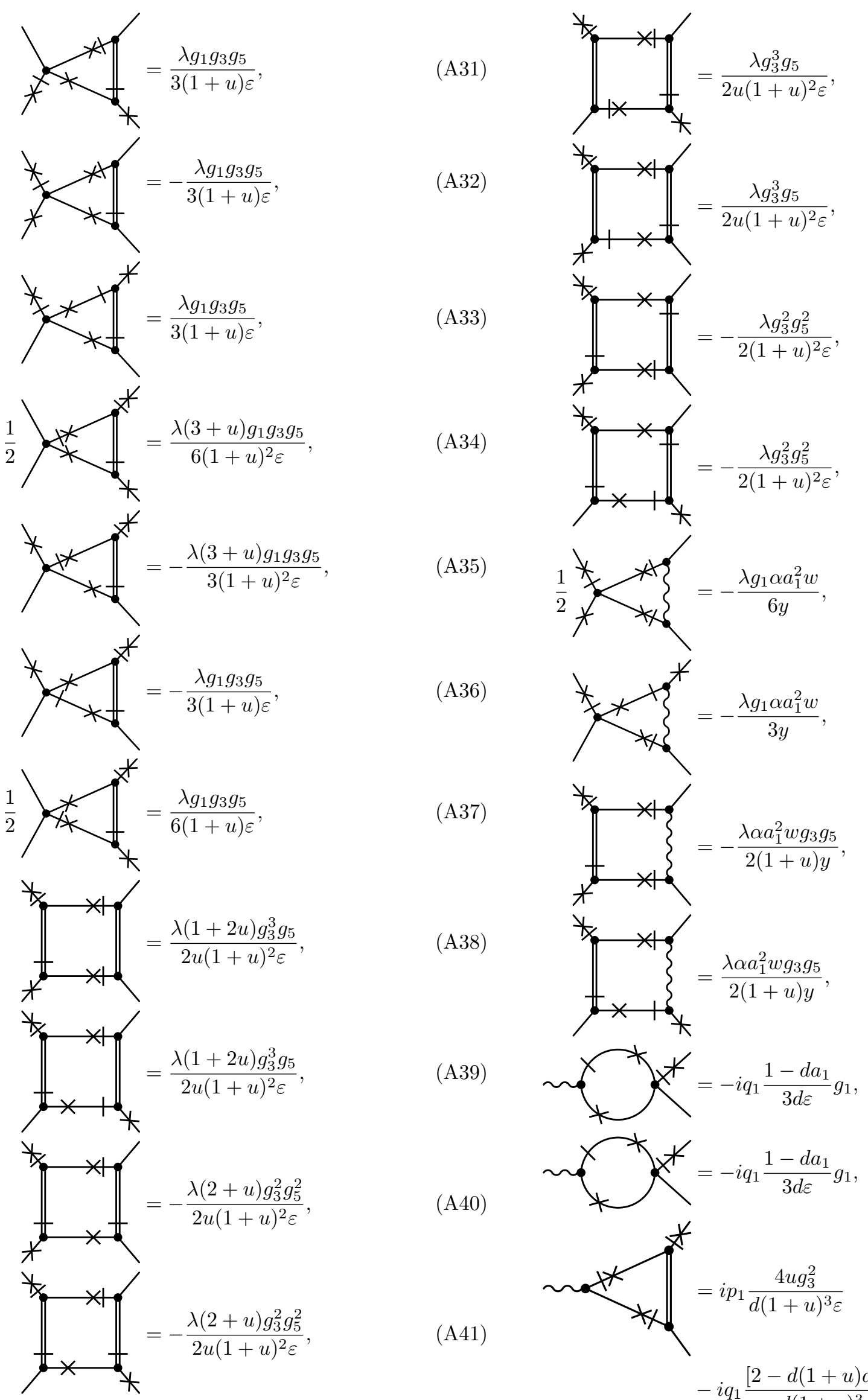

$$
-i q_{1} \frac{\left[2-d(1+u) a_{1}\right] g_{3}^{2}}{d(1+u)^{3} \varepsilon}
$$




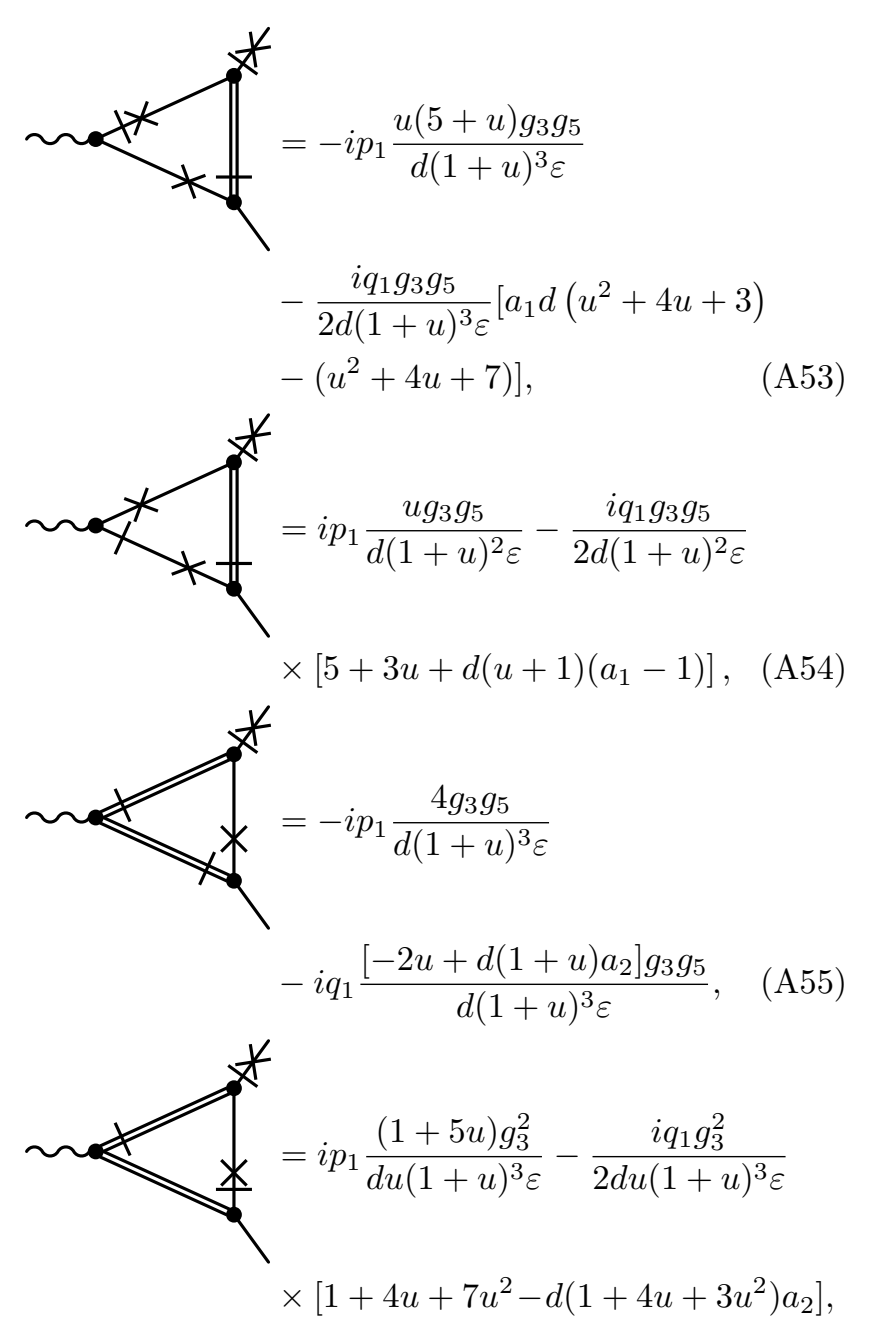

(A56)

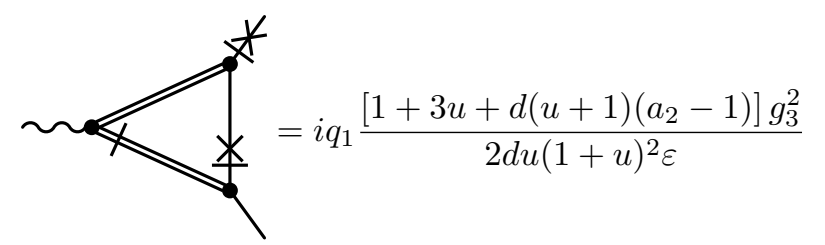

$-i p_{1} \frac{g_{3}^{2}}{d u(1+u)^{2} \varepsilon}$,

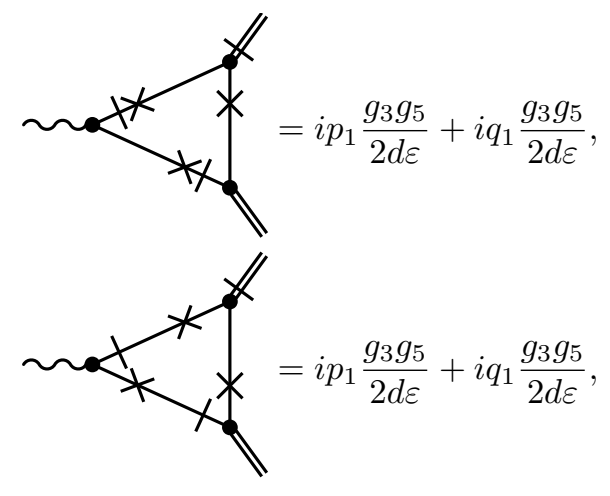

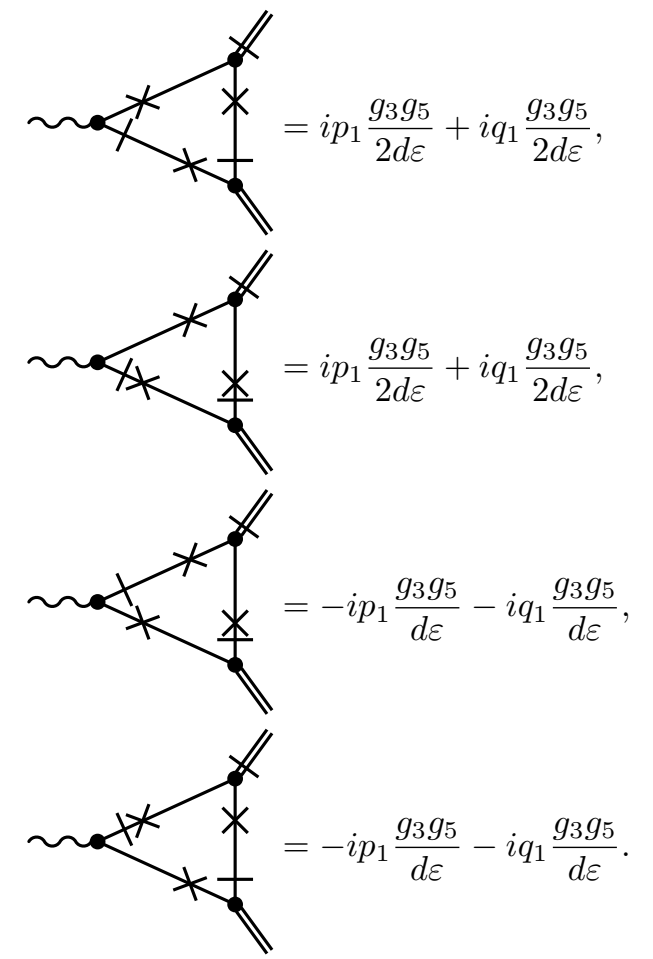

\section{Appendix B: Anomalous dimensions to the one-loop order}

In this section, we review the explicit expressions for the anomalous dimension $\gamma_{x}, x \in\left\{g_{1}, g_{3}, g_{5}, u, w, a_{1}, a_{2}\right\}$ of the charges and for the fields $x \in\left\{\psi, \psi^{\prime}, \boldsymbol{v}\right\}$, respectively. From relations 61)- 65), the following expressions directly follow

$$
\begin{aligned}
\gamma_{\lambda} & =-\gamma_{w}=\frac{4 g_{3}^{2}}{d(1+u)^{3}}+\frac{g_{3} g_{5}[d-4+d u(2+u)]}{d(1+u)^{3}} \\
& +\frac{w(d-1+\alpha)}{2 d}, \\
\gamma_{u} & =-\frac{4 g_{3}^{2}}{d(1+u)^{3}}+\frac{w(d-1+\alpha)(1-u)}{2 d u} \\
& -\frac{g_{3} g_{5}\left[2 u^{3}-u^{2}(d-6)-2 u(d-1)+2-d\right]}{d u(1+u)^{3}}, \quad(\mathrm{~B} 2) \\
\gamma_{g_{3}} & =-\frac{4 g_{3}^{2}}{d(1+u)^{3}}+\frac{g_{5}^{2}}{d u}-\frac{w}{2}\left(1-\frac{1-\alpha}{d}-\frac{2 a_{1} a_{2} \alpha}{1+u}\right) \\
& +\frac{g_{3} g_{5}\left[2\left(u^{3}+3 u^{2}+7 u+1\right)-d(1+u)^{2}(1+3 u)\right]}{2 d u(1+u)^{3}}, \\
\gamma_{g_{5}} & =\frac{2 g_{3}^{2}\left[d\left(2+3 u+u^{2}\right)-6-3 u-u^{2}\right]}{d(1+u)^{3}} \\
& +\frac{w}{d}\left[1-d+\alpha\left(\frac{d-2}{2}+a_{1}\left(a_{1}-1\right)(d-1)\right)\right] \\
& +\frac{g_{3} g_{5}}{2 d u(1+u)^{3}}\left[2\left(u^{3}+3 u^{2}+9 u-1\right)\right. \\
& \left.-d\left(5 u^{3}+13 u^{2}+7 u-1\right)\right]-\frac{g_{5}^{2}}{d u},
\end{aligned}
$$




$$
\begin{aligned}
\gamma_{g_{1}} & =\frac{2 g_{3}\left[d\left(2+3 u+u^{2}\right)-4\right]\left(g_{3}-g_{5}\right)}{d(1+u)^{3}}-\frac{6 g_{3}^{2} g_{5}\left(g_{3}-g_{5}\right)}{u g_{1}(1+u)} \\
& -\frac{5 g_{1}}{3}+\frac{w}{d}\left[1-d+\alpha\left(\frac{d}{2}-1+d a_{1}\left(2 a_{1}-1\right)\right)\right], \\
\gamma_{m} & =-\gamma_{m^{\prime}}=\frac{g_{3} g_{5}(d-2)}{2 d u}-\frac{g_{5}^{2}}{d u}, \\
\gamma_{\psi} & =\gamma_{\psi^{\dagger}}=\frac{g_{3}\left(g_{5}-g_{3}\right)\left[d\left(3+4 u+u^{2}\right)-4\right]}{2 d(1+u)^{3}} \\
& +\frac{w}{4 d}\left[d-1-\alpha\left(d\left(a_{1}-1\right)^{2}-1\right)\right], \\
\gamma_{\psi^{\prime}} & =\gamma_{\psi^{\dagger}}=\frac{g_{3}\left(g_{5}-g_{3}\right)\left[4-d(1+u)^{2}\right]}{2 d(1+u)^{3}} \\
& +\frac{w}{4 d}\left[1-d+\alpha\left(d\left(a_{1}-1\right)^{2}-1\right)\right], \\
\gamma_{a_{1}} & =\frac{g_{1}\left(1-d a_{1}\right)}{6 a_{1}}+g_{3} g_{5} \frac{d(2+u)-4}{4(1+u)^{2}} \\
& +g_{3}^{2} \frac{4 u\left(1+2 a_{1}\right)+d\left[1+u-2 a_{1} u-2 a_{2}(1+2 u)\right]}{8 u(1+u)^{2} a_{1}}, \\
& +\frac{g_{3} g_{5}}{8(1+u)^{2} a_{1}}\left[d\left(2 a_{2}-1-u\right)\right. \\
& +2(u-1)+2 a_{1}[d(2+u)-4], \\
\gamma_{a_{2}} & =0 . \\
& \text { A } 20
\end{aligned}
$$

\section{Appendix C: Coordinates of fixed points}

In this section we list coordinates of all fixed points for model $\mathrm{E}$ with compressible velocity fluctuations. The expression "not fixed" (NF) stands for a situation when a given fixed point coordinate can not be unambiguously determined from a solution to RG flow equations (71).

\begin{tabular}{|c|c|c|c|c|c|c|}
\hline FP FP1 & FP2 & FP3 & FP4 & FP5 & FP6 \\
\hline$g_{1}$ & 0 & $\frac{3 \varepsilon}{5}$ & 0 & $\frac{3}{5} \varepsilon$ & 0 & 0 \\
\hline$g_{3}$ & 0 & 0 & $\sqrt{\varepsilon}$ & $\sqrt{\varepsilon}$ & 0 & 0 \\
\hline$g_{5}$ & 0 & 0 & $\sqrt{\varepsilon}$ & $\sqrt{\varepsilon}$ & 0 & $\sqrt{2 \varepsilon+\frac{8\left[\alpha-3+3 a_{1} \alpha\left(a_{1}-1\right)\right]}{\alpha+3} y}$ \\
\hline$w$ & 0 & 0 & 0 & 0 & $\frac{8 y}{3+\alpha}$ & $\frac{8 y}{3+\alpha}$ \\
\hline$u$ & $\mathrm{NF}$ & $\mathrm{NF}$ & 1 & 1 & 1 & 1 \\
\hline$a_{1}$ & $\mathrm{NF}$ & $\frac{1}{4}$ & $a_{2}-\frac{1}{4}$ & $5 a_{2}-\frac{9}{4}$ & $\mathrm{NF}$ & $\mathrm{NF}$ \\
\hline
\end{tabular}

TABLE III: Coordinates of fixed points FP1 up to FP6.

Fixed point's value of the charge $a_{2}$ is in general not fixed, only for FP3 and FP4 there is an aforementioned relationship between $a_{1}^{*}$ and $a_{2}^{*}$.

\section{Appendix D: Eigenvalues of $\Omega$-matrix}

In this section we list all eigenvalues for fixed points

\begin{tabular}{|c|c|c|}
\hline FP & FP7 & FP8 \\
\hline$g_{1}$ & $\frac{3}{5}\left[\varepsilon+\frac{\alpha-6}{3+\alpha} y\right]$ & $\frac{3}{5}\left[\varepsilon+\frac{\alpha-6}{3+\alpha} y\right]$ \\
\hline$g_{3}$ & 0 & 0 \\
\hline$g_{5}$ & 0 & $\sqrt{2 \varepsilon+\frac{7 \alpha-48}{6+2 \alpha} y}$ \\
\hline$w$ & $\frac{8 y}{3+\alpha}$ & $\frac{8 y}{3+\alpha}$ \\
\hline$u$ & 1 & 1 \\
\hline$a_{1}$ & $\frac{1}{4}$ & $\frac{1}{4}$ \\
\hline
\end{tabular}

TABLE IV: Coordinates of fixed points FP7 and FP8.

from App. C.

\begin{tabular}{|c|c|c|c|c|}
\hline FP1 & FP2 & FP3 & FP4 & FP5 \\
\hline$-\varepsilon$ & $\varepsilon$ & $-\varepsilon$ & $-\frac{\varepsilon}{10}$ & $2 y \frac{3-\alpha+3 a_{1} \alpha\left(1-a_{1}\right)}{3+\alpha}-\frac{\varepsilon}{2}$ \\
\hline$-y$ & $-\frac{\varepsilon}{2}$ & $-\frac{\varepsilon}{2}$ & $\frac{\varepsilon}{4}$ & $2 y \frac{3-\alpha+4 a_{1} \alpha\left(1-2 a_{1}\right)}{3+\alpha}-\varepsilon$ \\
\hline$-\frac{\varepsilon}{2}$ & $-\frac{\varepsilon}{2}$ & $\frac{\varepsilon}{4}$ & $\varepsilon$ & $\frac{3+\alpha-4 a_{1} a_{2} \alpha}{3+\alpha}-\frac{\varepsilon}{2}$ \\
\hline$-\frac{\varepsilon}{2}$ & $\frac{2}{5} \varepsilon$ & $\varepsilon$ & $\varepsilon$ & $y$ \\
\hline 0 & $-y$ & $\frac{3 \varepsilon}{2}$ & $\frac{3 \varepsilon}{2}$ & $y$ \\
\hline 0 & 0 & $\frac{\varepsilon}{2}-y$ & $\frac{\varepsilon}{2}-y$ & 0 \\
\hline
\end{tabular}

TABLE V: Eigenvalues of matrix $\Omega$ (see Eq. 72 ) for IR stable fixed points corresponding to regimes FP1 up to FP5.

\begin{tabular}{|c|c|c|}
\hline FP6 & FP7 & FP8 \\
\hline$\varepsilon+4 y \frac{\alpha-3+3 a_{1} \alpha\left(a_{1}-1\right)}{3+\alpha}$ & $y$ & $y$ \\
\hline $2 y \frac{3-\alpha+4 a_{1} \alpha\left(1-2 a_{1}\right)}{3+\alpha}-\varepsilon$ & $y$ & $y$ \\
\hline$\frac{9-\alpha+6 a_{1} \alpha\left(1-a_{1}\right)-4 a_{1} a_{2} \alpha}{3+\alpha}-\varepsilon$ & $\frac{2}{5}\left(\varepsilon+y \frac{\alpha-6}{3+\alpha}\right)$ & $\frac{2}{5}\left(\varepsilon+y \frac{\alpha-6}{3+\alpha}\right)$ \\
\hline$y$ & $\varepsilon+y \frac{\alpha-6}{3+\alpha}$ & $\varepsilon+y \frac{\alpha-6}{3+\alpha}$ \\
\hline$y$ & $\frac{1}{8}\left(\frac{48-7 \alpha}{3+\alpha} y-4 \varepsilon\right)$ & $\frac{7 \alpha-48}{4(3+\alpha)} y+\varepsilon$ \\
\hline 0 & $y-\frac{a_{2} \alpha y}{3+\alpha}-\frac{\varepsilon}{2}$ & $y \frac{72+\alpha-8 a_{2} \alpha}{8(3+\alpha)}-\varepsilon$ \\
\hline
\end{tabular}

TABLE VI: Eigenvalues of matrix $\Omega$ (see Eq. 72 ) for IR stable fixed points corresponding to regimes FP6 up to FP8. 
[1] U. Täuber, Critical Dynamics: A Field Theory Approach to Equilibrium and Non-Equilibrium Scaling Behavior (Cambridge University Press, New York, 2014).

[2] M. Henkel, H. Hinrichsen, and S. Lübeck, Nonequilibrium phase transitions: Volume 1 Absorbing phase transitions (Springer, Dordrecht, 2008).

[3] M. A. Muñoz, Rev. Mod. Phys. 90, 031001 (2018).

[4] D. ben Avraham and S. Havlin, Diffusion and Reactions in Fractals and Disordered Systems (Cambridge University Press, 2000).

[5] B. B. Mandelbrot, Fractals and Scaling in Finance (Springer, 1997)

[6] P. L. Krapivsky, S. Redner, and E. Ben-Naim, A Kinetic View of Statistical Physics (Cambridge University Press, 2010).

[7] R. Pastor-Satorras, C. Castellano, P. Van Mieghem, and A. Vespignani, Rev. Mod. Phys. 87, 925 (2015).

[8] P. C. Hohenberg and B. I. Halperin, Rev. Mod. Phys. 49, 435 (1977).

[9] R. Folk and G. Moser, J. Phys. A: Math. Gen. 39, R207 (2006).

[10] A. N. Vasil'ev, The Field Theoretic Renormalization Group in Critical Behavior Theory and Stochastic Dynamics (Chapman\&Hall/CRC Press, New York, 2004).

[11] G. F. Mazenko, Nonequilibrium Statistical Mechanics (Wiley-VCH, Weinheim, 2006).

[12] V. Dohm, Phys. Rev. B 73, 092503 (2006).

[13] M. Dančo, M. Hnatič, M. V. Komarova, T. Lučivjanský, and M. Y. Nalimov, Phys. Rev. E 93, 012109 (2016).

[14] Y. A. Zhavoronkov, M. V. Komarova, Y. G. Molotkov, M. Y. Nalimov, and J. Honkonen, Theor. Math. Phys. 200, 1237 (2019).

[15] M. Hnatich, M. V. Komarova, and M. Y. Nalimov, Theor. Math. Phys. 175, 779 (2013).

[16] C. DeDominicis and L. Peliti, Phys. Rev. Lett. 38, 505 (1977).

[17] C. DeDominicis and L. Peliti, Phys. Rev. B 18, 353 (1978).

[18] V. Dohm, Z. Phys. B 33, 79 (1979).

[19] L. T. Adzhemyan, M. Dančo, M. Hnatič, E. V. Ivanova, and M. V. Kompaniets, EPJ Web Conf. 108, 02004 (2016).

[20] M. V. Komarova, D. M. Krasnov, and M. Y. Nalimov, Theor. Math. Phys. 169, 89 (2011).

[21] M. Dančo, M. Hnatich, M. V. Komarova, D. M. Krasnov, T. Lučivjanský, L. Mižišin, and M. Y. Nalimov, Theor. Math. Phys. 176, 888 (2013).

[22] U. Frisch, Turbulence: The Legacy of A. N. Kolmogorov (Cambridge University Press, Cambridge, 1995).

[23] L. T. Adzhemyan, N. V. Antonov, and A. N. Vasil'ev, The Field Theoretic Renormalization Group in Fully Developed Turbulence (Gordon \& Breach, London, 1999).

[24] G. Falkovich, K. Gawedzki, and M. Vergassola, Rev. Mod. Phys. 73, 913 (2001).

[25] D. Y. Ivanov, Critical Behavior of Non-Idealized Systems (Fizmatlit, Moscow, 2003).

[26] D. E. Khmelnitski, Sov. Phys. JETP 41, 981 (1975).

[27] H. K. Janssen, K. Oerding, and E. Sengespeick, J. Phys.
A: Math. Gen. 28, 6073 (1995).

[28] G. Satten and D. Ronis, Phys. Rev. Lett. 55, 91 (1985).

[29] G. Satten and D. Ronis, Phys. Rev. A 33, 3415 (1986).

[30] N. V. Antonov, J. Phys. A: Math. Gen 39, 7825 (2006).

[31] R. H. Kraichnan, Phys. Fluids 11, 945 (1968).

[32] N. V. Antonov, Phys. Rev. E 60, 6691 (1999).

[33] L. T. Adzhemyan, N. V. Antonov, and J. Honkonen, Phys. Rev. E 66, 036313 (2002).

[34] N. V. Antonov, Physica D 144, 370 (2000).

[35] N. V. Antonov, M. Hnatich, J. Honkonen, and M. Jurcisin, Phys. Rev. E 68, 046306 (2003).

[36] M. Chertkov, G. Falkovich, and V. Lebedev, Phys. Rev. Lett. 76, 3707 (1996).

[37] G. Eyink, Phys. Rev. E 54, 1497 (1996).

[38] M. Vergassola and M. Avellaneda, Physica D 106, 148 (1997).

[39] L. T. Adzhemyan and N. V. Antonov, Phys. Rev. E 58, 7381 (1998).

[40] J. Honkonen, Theor. Math. Phys. 175, 827 (2013).

[41] J. Honkonen, M. V. Komarova, Y. G. Molotkov, and M. Y. Nalimov, Nucl. Phys. B. 939, 105 (2019).

[42] A. N. Vasil'ev, Functional Methods in Quantum Field Theory and Statistical Physics (Gordon and Breach Science Publishers, Amsterdam, 1998).

[43] L. D. Landau and E. M. Lifshitz, Fluid Mechanics (Pergamon Press, 1959).

[44] J. Zinn-Justin, Quantum Field Theory and Critical Phenomena (4th edition, Oxford University Press, Oxford, 2002).

[45] H. K. Janssen, Z. Phys. B 23, 377 (1976).

[46] C. D. Dominicis, J. Phys. (Paris), Suppl C1 37, 247 (1976).

[47] P. C. Martin, E. D. Siggia, and H. A. Rose, Phys. Rev. A 8, 423 (1973).

[48] N. V. Antonov and A. S. Kapustin, J. Phys. A: Math. Theor. 43, 405001 (2010).

[49] K. Gawedzki and M. Vergassola, Physica D 138, 63 (2000).

[50] D. J. Amit and V. Martín-Mayor, Field Theory, the Renormalization Group and Critical Phenomena (World Scientific, Singapore, 2005).

[51] A. Weinrib and B. I. Halperin, Phys. Rev. B 27, 413 (1983).

[52] G. Satten and D. Ronis, Phys. Rev. A 33, 3415 (1986).

[53] D. Y. Volchenkov and M. Y. Nalimov, Theor. Math. Phys. 106, 375 (1996).

[54] L. T. Adzhemyan, M. Hnatich, and J. Honkonen, Eur. Phys. J. B 73, 275 (2010).

[55] J. Honkonen, Chaotic Modeling and Simulation Journal 2, 139 (2018), URL http://www.cmsim.eu/papers_pdf/ april_2018_papers/April_2_2018_CMSIM_Honkonen_ 139-158.pdf

[56] K. J. Wiese, Phys. Rev. E 56, 5013 (1997).

[57] N. V. Antonov, M. Y. Nalimov, and A. A. Udalov, Theor. Math. Phys. 110, 385 (1997).

[58] N. V. Antonov, N. M. Gulitskiy, M. M. Kostenko, and T. Lučivjanský, Phys. Rev. E 95, 033120 (2017). 